\title{
Pacific
}

Journal of

Mathematics

CERTAIN $C^{*}$-ALGEBRAS WITH REAL RANK ZERO AND THEIR CORONA AND MULTIPLIER ALGEBRAS. PART I

SHUANG ZHANG

Volume $155 \quad$ No. 1 


\section{CERTAIN $C^{*}$-ALGEBRAS \\ WITH REAL RANK ZERO AND THEIR CORONA AND MULTIPLIER ALGEBRAS. PART I}

\section{SHUANG ZHANG}

We first prove that every $\sigma$-unital, purely infinite, simple $C^{*}$ algebra is either unital or stable. Consequently, purely infinite simple $C^{*}$-algebras have real rank zero. In particular, the Cuntz algebras $\mathscr{O}_{n}$ $(2 \leq n \leq+\infty)$ and the Cuntz-Krieger algebras $\mathscr{O}_{A}$, where $A$ can be any irreducible matrix, contain abundant projections. This includes an answer for a question raised by $B$. Blackadar in $[5,2.10]$. We then prove that the corona and multiplier algebras associated with many interesting $C^{*}$-algebras have real rank zero. As special cases, we consider the multiplier and corona algebras associated with certain simple $\mathrm{AF}$ algebras, the stabilizations of type $\mathrm{II}_{1}$ and type $\mathrm{III}$ factors, the Cuntz algebras and certain Cuntz-Krieger algebras, the BunceDeddens algebras and some irrational rotation algebras. $A$ recent result of $L$. G. Brown and G. K. Pedersen in [12, 3.21] is included as a special case. In particular, $K_{1}(\mathscr{A})=0$, where $\mathscr{A}$ is a $\sigma$-unital, purely infinite simple $C^{*}$-algebra, if and only if the generalized Weylvon Neumann theorem holds in $M(\mathscr{A} \otimes \mathscr{K})$.

Introduction. $\mathscr{L}(\mathscr{H})$ and $\mathscr{K}$ denote the algebra of all bounded operators and the algebra of all compact operators on a separable Hilbert space $\mathscr{H}$, respectively. The well-known classical Weyl-von Neumann theorem asserts that every self-adjoint operator in $\mathscr{L}(\mathscr{H})$ can be written as a sum of a bounded self-adjoint diagonal operator and a compact operator. If $\mathscr{H}$ is replaced by a Hilbert $C^{*}$-module $\mathscr{H}_{\mathscr{A}}$ associated with a $C^{*}$-algebra $\mathscr{A}$, we denote the $C^{*}$-algebra of bounded operators on $\mathscr{H}_{\mathscr{A}}$ whose adjoint exists by $\mathscr{L}\left(\mathscr{H}_{\mathscr{A}}\right)$ and denote the norm closure of operators of 'finite rank' by $\mathscr{K}_{(}\left(\mathscr{H}_{\mathscr{A}}\right)$. Then $\mathscr{K}\left(\mathscr{H}_{\mathscr{A}}\right)$ is a closed ideal of $\mathscr{L}\left(\mathscr{H}_{\mathscr{A}}\right)$,

$\mathscr{L}\left(\mathscr{H}_{\mathscr{A}}\right) \cong M(\mathscr{A} \otimes \mathscr{K})$ and $\mathscr{K}\left(\mathscr{H}_{\mathscr{A}}\right) \cong \mathscr{A} \otimes \mathscr{K}$ as $C^{*}$-algebras,

(see [23]). It is desirable to see whether a generalized version of the Weyl-von Neumann theorem still holds in $\mathscr{L}\left(\mathscr{H}_{\mathscr{A}}\right)$. This problem can be more generally formulated in the multiplier algebra $M(\mathscr{B})$ of a non-unital $C^{*}$-algebra $\mathscr{B}$ (may not be stable). Even more general, one can consider a similar problem in certain $C^{*}$-algebras with respect to their closed ideals $([26])$. We say that a generalized 
Weyl-von Neumann theorem holds in $M(\mathscr{B})$, if every self-adjoint element $x$ of $M(\mathscr{B})$ can be written in the form:

$$
x=\sum_{i=1}^{\infty} \lambda_{i} p_{i}+a, \quad \text { where } \sum_{i=1}^{\infty} p_{i}=1 \text { and }\left\{\lambda_{i}\right\} \in l^{\infty}(R)
$$

i.e., a sum of a self-adjoint element $a$ of $\mathscr{B}$ and a diagonal selfadjoint element $\sum_{i=1}^{\infty} \lambda_{i} p_{i}$ of $M(\mathscr{B})$ with respect to a sequence of mutually orthogonal projections $\left\{p_{i}\right\}$ of $\mathscr{B}$ ( $x$ is said to be quasidiagonal). The structure of $M(\mathscr{B})$ would be clear if the generalized Weyl-von Neumann theorem holds. However, many aspects of the multiplier algebras of simple AF algebras have not been well understood yet.

It was pointed out $([37, \S 3])$ that the generalized Weyl-von Neumann theorem holds in $M(\mathscr{B})$ if and only if $M(\mathscr{B})$ has the (FS) property: Self-adjoint elements with finite spectrum is norm dense in the set of all self-adjoint elements. Hence, if the Weyl-von Neumann theorem holds in $M(\mathscr{B})$, then $\mathscr{B}$, as a closed ideal of $M(\mathscr{B})$, must have the (FS) property. Moreover, ' $K_{1}(\mathscr{B})=0$ ' is one additional necessary condition in case $\mathscr{B}$ is stable $([37,3.2])$. Recently, L. G. Brown and G. K. Pedersen proved $([12,2.6])$ that a $C^{*}$-algebra $\mathscr{A}$ has the (FS) property if and only if $\mathscr{A}$ has real rank zero (i.e., $R R(\mathscr{A})=0$ ); and again if and only if $R R(\mathscr{A} \otimes \mathscr{K})=0$. Hence, the generalized Weylvon Neumann theorem holds in $M(\mathscr{B})$ if and only if $R R(M(\mathscr{B}))=$ 0 .

In this article we will be concerned with $\sigma$-unital, simple $C^{*}$-algebras of real rank zero. In the first section, we give a structural description for purely infinite, simple $C^{*}$-algebras. We prove, Theorem (1.2), that every $\sigma$-unital, purely infinite, simple $C^{*}$-algebra is either stable or unital. Consequently, every purely infinite, simple $C^{*}$-algebra has the (FS) property, and hence has real rank zero by [12, 2.6]. As a by-product, the above general result includes an answer for a question raised by B. Blackadar in [5, 2.10] concerning the structure of projections in the Cuntz algebras $\mathscr{O}_{n}(2 \leq n \leq+\infty)$. The motivation of the above description comes from two aspects: one is the importance of purely infinite, simple $C^{*}$-algebras in the theory of simple $C^{*}$-algebras in which many problems are still open. For example, it is even not known whether the direct sum of two finite projections is still finite. Another is that the corona algebras of many simple $C^{*}$-algebras, and hence their nonzero hereditary $C^{*}$-subalgebras, are purely infinite and simple. 
In the second section, we consider $C^{*}$-algebras whose corona algebras are either simple or have a unique nontrivial closed ideal. First, if $M(\mathscr{A}) / \mathscr{A}$ is simple, then $M(\mathscr{A}) / \mathscr{A}$ is purely infinite by [38, $\S 1]$. It follows from Theorem (1.2) that $R R(M(\mathscr{A}) / \mathscr{A})=0$. Then using the lifting from the corona algebra to the multiplier algebra we conclude that $R R(M(\mathscr{A}))=0$ if and only if every projection in $M(\mathscr{A}) / \mathscr{A}$ lifts to a projection in $M(\mathscr{A})$ (this condition is equivalent to ' $K_{1}(\mathscr{A})=0$ ' in case $\left.\mathscr{A} \cong \mathscr{A} \otimes \mathscr{K}[37, \S 2]\right)$. In particular, in case $\mathscr{A}$ is a $\sigma$-unital, purely infinite, simple $C^{*}$-algebra, we conclude that $R R(M(\mathscr{A} \otimes \mathscr{K}) / \mathscr{A} \otimes \mathscr{K})=0([40,3.3])$, and that $R R(M(\mathscr{A} \otimes \mathscr{K}))=0$ if and only if $K_{1}(\mathscr{A})=0$. More specifically, if $\mathscr{A}$ is a type III factor, or if $\mathscr{A}=\mathscr{O}_{n}(2 \leq n \leq+\infty)$, then $R R(M(\mathscr{A} \otimes \mathscr{K}))=0$. But the generalized Weyl-von Neumann theorem does not hold in $M(\mathscr{A} \otimes \mathscr{K})$ if $\mathscr{A}$ is the Calkin algebra or if $\mathscr{A}=\mathscr{O}_{A}$ where $\operatorname{det}(I-A)=0$ and $A$ is an irreducible matrix (since $K_{1}(\mathscr{A}) \neq 0[15,4.5]$ ). If $\mathscr{A}$ is a separable, non-unital, simple AF algebra with continuous scale (such a $C^{*}$-algebra is 'very finite' [24]), in particular if $\mathscr{A}$ is a nonunital, finite matroid algebra, then $R R(M(\mathscr{A}))=0$. Secondly, if there is only one nontrivial closed ideal $\mathscr{J}_{0}$ between $\mathscr{A} \otimes \mathscr{K}$ and $M(\mathscr{A} \otimes \mathscr{K})$, we prove that both $M(\mathscr{A} \otimes \mathscr{K}) / \mathscr{J}_{0}$ and $\mathscr{J}_{0} / \mathscr{A} \otimes \mathscr{K}$ are purely infinite and simple, and hence $R R\left(M(\mathscr{A} \otimes \mathscr{K}) / \mathscr{J}_{0}\right)=R R\left(\mathscr{J}_{0} / \mathscr{A} \otimes \mathscr{K}\right)=0$. Using the lifting again, we conclude that $R R(M(\mathscr{A} \otimes \mathscr{K}))=0$ if $K_{1}(\mathscr{A})=K_{1}\left(\mathscr{L}_{0}\right)=0$. This result has covered many interesting $C^{*}$ algebras with real rank zero. For example, if $\mathscr{A}$ is a simple AF algebra with a unique trace (up to multiples), in particular if $\mathscr{A}$ is a separable matroid algebra, then $R R(M(\mathscr{A} \otimes \mathscr{K}))=0$. (The above two general results have included [12, 3.21].) The same conclusion holds if $\mathscr{A}$ is a type $\mathrm{II}_{1}$ factor. If $\mathscr{A}$ is a Bunce-Deddens algebra, then $R R(M(\mathscr{A} \otimes \mathscr{K}) / \mathscr{A} \otimes \mathscr{K})=0$ but $R R(M(\mathscr{A} \otimes \mathscr{K})) \neq 0$, where $M(\mathscr{A} \otimes \mathscr{K}) / \mathscr{A} \otimes \mathscr{K}$ has a unique nontrivial closed ideal. If an irrational rotation algebra $\mathscr{A}_{\theta}$ has real rank zero (at least a dense subset of irrational numbers satisfy this condition, see [14]), then $M\left(\mathscr{A}_{\theta} \otimes \mathscr{K}\right) / \mathscr{A}_{\theta} \otimes \mathscr{K}$ has a unique nontrivial closed ideal defined by the unique trace on $\mathscr{A}_{\theta}$. Since $K_{1}\left(\mathscr{A}_{\theta}\right) \neq 0$, it follows that $R R\left(M\left(\mathscr{A}_{\theta} \otimes \mathscr{K}\right)\right) \neq 0$.

In the third section we first point out that the classical Weyl-von Neumann theorem holds if and only if $K_{1}(\mathscr{K})=0$ and $R R(\mathscr{K})=0$. The proof involves only $C^{*}$-algebraic techniques, neither using the fact that $\mathscr{L}(\mathscr{H})$ is a von Neumann algebra nor using any techniques arising from the underline space $\mathscr{H}$ at all. This provides new insights into the Weyl-von Neumann theorem. Secondly, we give a full 
description for all hereditary $C^{*}$-subalgebras of $M(\mathscr{A} \otimes \mathscr{K})$ in case $\mathscr{A}$ is purely infinite and simple. As a consequence, $K_{1}(\mathscr{A})=0$ if and only if every essentially unital, non-unital, hereditary $C^{*}$-subalgebra of $M(\mathscr{A} \otimes \mathscr{K})$ has an approximate identity consisting of projections. In addition, we provide a way to construct a sequence of purely infinite simple corona algebras starting with one purely infinite simple $C^{*}$-algebra.

In the fourth section, we prove that if $\mathscr{A}$ is a purely infinite, simple (not necessarily unital) $C^{*}$-algebra, and $p$ is an infinite projection of $\mathscr{A}$, then $K_{1}(\mathscr{A})$ is isomorphic to the group $U(p \mathscr{A} p) / U_{0}(p \mathscr{A} p)$, where $U(p \mathscr{A} p)$ is the unitary group of $p \mathscr{A} p$ and $U_{0}(p \mathscr{A} p)$ is the path component of $U(p \mathscr{A} p)$ containing its identity $p$. This slightly extending a result of [15] where $\mathscr{A}$ is assumed to be a unital, purely infinite, simple $C^{*}$-algebra.

All notations in this paper are standard. We shall denote the Murrayvon Neumann equivalence of two projections $p$ and $q$ in a $C^{*}$ algebra $\mathscr{A}$ by ' $p \sim q$ ' (i.e., there exists a partial isometry $v$ in $\mathscr{A}$ such that $v v^{*}=p$ and $v^{*} v=q$ ). ' $p \lesssim q$ ' means that $p$ is equivalent to a subprojection of $q$. ' $\lesssim$ ' naturally induces a partial order ' $\leq$ ' on the semigroup of equivalence classes of projections in $\mathscr{A}$, denoted by $D(\mathscr{A})$. The equivalence class of a projection $p$ is denoted by $[p] . \quad\left\{e_{i j}: i, j \geq 1\right\}$ denotes the set of matrix units of $\mathscr{K}$. We reserve the notation $\pi$ for the canonical quotient map from $M(\mathscr{A})$ to $M(\mathscr{A}) / \mathscr{A}$.

1. Purely infinite, simple $C^{*}$-algebras. A $C^{*}$-subalgebra $\mathscr{B}$ of a $C^{*}$-algebra $\mathscr{A}$ is said to be hereditary if ' $0 \leq a \leq b \in \mathscr{B}$ and $b \in$ $\mathscr{A}^{\prime}$ ' implies ' $a \in \mathscr{B}$ '. An equivalent condition is that $\mathscr{B}=\mathscr{L} \cap$ $\mathscr{L}^{*}$, where $\mathscr{L}$ is a closed left ideal of $\mathscr{A}([29,1.5 .2])$. Another equivalent condition is that $(\mathscr{B} \mathscr{A} \mathscr{B})^{-}=\mathscr{B}$, where $(\mathscr{B} \mathscr{A} \mathscr{B})^{-}$is the $C^{*}$-subalgebra of $\mathscr{A}$ generated by those elements with the form $b_{1} a b_{2}$ with $b_{1}, b_{2} \in \mathscr{B}$ and $a \in \mathscr{A}$. In particular, every closed ideal is a hereditary $C^{*}$-subalgebra. We denote the hereditary $C^{*}$-subalgebra $\left(x \mathscr{A} x^{*}\right)^{-}$of $\mathscr{A}$ generated by a nonzero element $x$ of $M(\mathscr{A})$ by $\mathscr{A}_{x}$ or $\operatorname{her}(x)$.

A $C^{*}$-algebra $\mathscr{A}$ is said to be purely infinite, if every nonzero hereditary $C^{*}$-subalgebra contains an infinite projection $([15,1.5]$ and $[38])$, where we call a projection infinite if it is Murray-von Neumann equivalent to a proper subprojection of itself. Equivalently, $\mathscr{A}$ is purely infinite if and only if $\mathscr{A}_{x}$ contains an infinite projection for any nonzero positive element $x$ of $\mathscr{A}$. 
Well-known examples of purely infinite simple $C^{*}$-algebras are the Calkin algebra and type III factors (all are nonseparable). Separable examples include the Cuntz algebras $\mathscr{O}_{n}(2 \leq n \leq \infty)$ and the Cuntz-Krieger algebras $\mathscr{O}_{A}$, if $A$ is an irreducible matrix ([15], [16] and [18]). We will see from this article that many corona algebras are also purely infinite and simple. Since purely infinite simple $C^{*}$ algebras, especially the Cuntz algebras, have proved their importance in the theory of simple $C^{*}$-algebras, of course further classification and description for these $C^{*}$-algebras are desirable. It turns out that such a $C^{*}$-algebra $\mathscr{A}$ either has a unit or is stable (i.e., $\mathscr{A} \cong \mathscr{A} \otimes \mathscr{K}$ ). As a consequence, purely infinite simple $C^{*}$-algebras have real rank zero, and hence contain abundant projections.

1.1. Lemma ([11, 4.23]). Suppose that $\mathscr{A}$ is a $\sigma$-unital $C^{*}$-algebra. If there exists a full projection $p$ in $M(\mathscr{A})$ such that $\mathscr{A}_{p}$ is stable, then there exists a partial isometry $u$ in $M(\mathscr{A})$ such that $u u^{*}=p$ and $u^{*} u=1$. Consequently, $\mathscr{A} \cong \mathscr{A} \otimes \mathscr{K}$.

Our main theorem in this section is stated as follows:

1.2. Theorem. (i) If $\mathscr{A}$ is a $\sigma$-unital, purely infinite, simple $C^{*}$ algebra, then either $\mathscr{A}$ is unital or $\mathscr{A}$ is stable. Consequently, $\mathscr{A}_{x}$ is either unital or stable for every nonzero positive element $x$ in $\mathscr{A}$.

(ii) Suppose that $\mathscr{A}$ is a simple $C^{*}$-algebra (may not be $\sigma$-unital). Then $\mathscr{A}$ is purely infinite if and only if $R R(\mathscr{A})=0$ and every nonzero projection of $\mathscr{A}$ is infinite.

Proof. (i) Suppose that $\mathscr{A}$ is not unital. By Lemma (1.1), it suffices to show that there exists a nonzero projection $p$ in $M(\mathscr{A})$ such that $\mathscr{A}_{p}$ is stable.

Let $a$ be a strictly positive element of $\mathscr{A}$ with $\|a\|=1$. Since $\mathscr{A}$ is not unital, 0 is a limit point of the spectrum $\sigma(a)$ of $a$. We define a sequence of continuous functions as follows:

$$
f_{n}(t)= \begin{cases}1 & \text { if } t \geq \varepsilon_{n}, \\ \frac{t-\varepsilon_{n+1}}{\varepsilon_{n}-\varepsilon_{n+1}} & \text { if } \varepsilon_{n+1} \leq t \leq \varepsilon_{n}, \\ 0 & \text { if } t \leq \varepsilon_{n+1}\end{cases}
$$

where $\varepsilon_{n} \searrow 0$. Set $e_{n}=f_{n}(a)$. Then $\left\{e_{n}\right\}$ is an approximate identity of $\mathscr{A}$ such that $e_{m} e_{n}=e_{n}$ if $m>n$. Consider her $\left(e_{2 n+2}-e_{2 n}\right)$ for 
$n \geq 1$. We can assume that $\operatorname{her}\left(e_{2 n+2}-e_{2 n}\right) \neq\{0\}$ for $n \geq 1$. Set $r_{n}=E_{\left(\varepsilon_{2 n+2}, \varepsilon_{2 n+1}\right)}\left(e_{2 n+2}\right)$, the spectral projection of $e_{2 n+2}$ in $\mathscr{A}^{* *}$ over the open interval $\left(\varepsilon_{2 n+2}, \varepsilon_{2 n+1}\right)$. Then $r_{n}$ is an open projection of $\mathscr{A}$ such that $\operatorname{her}\left(r_{n}\right) \subset \operatorname{her}\left(e_{2 n+2}-e_{2 n}\right)$. Moreover, it is easily verified by construction that $r_{n} r_{m}=0$ if $n \neq m$, and $r_{n}\left(e_{2 n+2}-e_{2 n}\right)=$ $\left(e_{2 n+2}-e_{2 n}\right) r_{n}=r_{n}$ for $n \geq 1$.

Since $\mathscr{A}$ is purely infinite, we can choose a nonzero projection $q_{n}$ in $\operatorname{her}\left(r_{n}\right)$ for $n \geq 1$ (of course, $q_{n}$ is infinite). Then $q_{n} \leq r_{n}$, and hence

$$
q_{n}\left(e_{2 n+2}-e_{2 n}\right)=q_{n} r_{n}\left(e_{2 n+2}-e_{2 n}\right)=q_{n} r_{n}=q_{n} \text {. }
$$

Similarly, $\left(e_{2 n+2}-e_{2 n}\right) q_{n}=q_{n}$. Since $\mathscr{A}$ is purely infinite and simple, using a routine argument for infinite simple $C^{*}$-algebra (see [5, 3.12.1], for example), we find a nonzero subprojection $p_{n}$ of $q_{n}$ such that $p_{n} \sim p_{1}=q_{1}$ for $n \geq 2$. Set

$$
p=\sum_{n=1}^{\infty} p_{n}
$$

Since $\left\{e_{2 n}\right\}$ is also an approximate identity of $\mathscr{A}$, it is routine that $p$ is a projection in $M(\mathscr{A})$. In fact, for every element $b$ in $\mathscr{A}$,

$$
\left\|b\left(\sum_{i=n}^{m} p_{i}\right) b^{*}\right\| \leq\left\|b\left[\sum_{i=n}^{m}\left(e_{2(i+1)}-e_{2 i}\right)\right] b^{*}\right\| \rightarrow 0
$$

if $n \rightarrow \infty$. It is obvious that $\mathscr{A}_{p}$ is stable. Since $\mathscr{A}$ is simple, Lemma (1.1) applies, and hence $\mathscr{A}$ is stable.

It is easy to check that $\mathscr{A}_{x}$ is purely infinite and simple for any nonzero positive element $x$ in $\mathscr{A}$. Hence, $\mathscr{A}_{x}$ either has a unit or $\mathscr{A}_{x} \cong \mathscr{A}_{x} \otimes \mathscr{K}$.

(ii) If $x$ is any nonzero positive element, then $\mathscr{A}_{x}$ is $\sigma$-unital. By (i), $\mathscr{A}_{x}$ is either unital or $\mathscr{A}_{x} \cong \mathscr{A}_{x} \otimes \mathscr{K}$. If $\mathscr{A}_{x}$ has a unit, then the unit is a projection of $\mathscr{A}$. If $\mathscr{A}_{x} \cong \mathscr{A}_{x} \otimes \mathscr{K}$, then $\mathscr{A}_{x} \cong p \mathscr{A}_{p} \otimes \mathscr{K}$ where $p$ is any nonzero projection of $\mathscr{A}_{x}([9,2.8])$, and hence $\mathscr{A}_{x}$ has an approximate identity of projections. It follows that $\mathscr{A}$ has the (HP) property, or equivalently has the (FS) property $([5,2.7]$ and [27]). Thus, $R R(\mathscr{A})=0$ by $[12,2.6]$. Of course, every nonzero projection of a purely infinite $C^{*}$-algebra is infinite. The direction 'if' is trivial. We have completed the proof.

We have the following corollary which includes an answer of the question [5, 2.10] raised by B. Blackadar. 
1.3. Corollary. The Cuntz algebras $\mathscr{O}_{n}(2 \leq n \leq \infty)$ and the Cuntz-Krieger algebras $\mathscr{O}_{A}$, where $A$ is any irreducible matrix, have the (FS) property, or equivalently have real rank zero.

Concerning the matrix algebras $M_{n}(\mathscr{A})$ over a purely infinite simple $C^{*}$-algebra, we have the following proposition:

1.4. Proposition. If $\mathscr{A}$ is a simple $C^{*}$-algebra (not necessarily $\sigma$-unital), then the following are equivalent:

(i) $\mathscr{A}$ is purely infinite.

(ii) $\mathscr{A} \otimes \mathscr{K}$ is purely infinite.

(iii) $M_{n}(\mathscr{A})$ is purely infinite for $n \geq 1$.

Proof. It is clear that (ii) implies (i) and (iii) implies (i).

If $\mathscr{A}$ is purely infinite and simple, then $R R(\mathscr{A})=0$ by Theorem (1.2). It follows from [12, 2.10] that $R R(\mathscr{A} \otimes \mathscr{K})=0$. Let $q$ be any nonzero projection of $\mathscr{A} \otimes \mathscr{K}$. Diagonalizing the projection $q$ by [39], there exists a unitary $u$ in $M(\mathscr{A} \otimes \mathscr{K})$ such that $u q u^{*}$ is a diagonal matrix with only finitely many nonzero diagonal entries which are nonzero projections of $\mathscr{A}$. Since every nonzero projection of $\mathscr{A}$ is infinite, hence $u q u^{*}$ is an infinite projection of $\mathscr{A} \otimes \mathscr{K}$. Thus it follows from Theorem (1.2) that $\mathscr{A} \otimes \mathscr{K}$ is purely infinite. Hence, (i) implies (ii). Similarly, (i) implies (iii).

1.5. Remarks. From Theorem (1.2)(i) we obtain some information on nonzero element $x$ of purely infinite simple $C^{*}$-algebra $\mathscr{A}$ by looking at the hereditary $C^{*}$-subalgebra $\mathscr{A}_{x}$. In case $\mathscr{A}_{x}$ has a unit $p$, there exists an element $y$ in $\mathscr{A}$ such that $x y x^{*}=p$. Hence the restriction of $x$ on its range is invertible. In case $\mathscr{A}_{x}$ is stable, then $\mathscr{A}_{x}$ contains a $C^{*}$-subalgebra which is ${ }^{*}$-isomorphic to $\mathscr{K}$.

In particular, we give a glance at the Calkin algebra $\mathscr{L}(\mathscr{H}) / \mathscr{K}$. It follows from the spectral decomposition theory for self-adjoint operators in $\mathscr{L}(\mathscr{H})$ that the Calkin algebra is purely infinite and simple. As a consequence of Theorem (1.2), the set of non-compact operators on $\mathscr{H}$ are divided into three classes. One is the set of operators such that $\mathscr{A}_{\bar{T}}=\mathscr{L}(\mathscr{H}) / \mathscr{K}$, i.e., the set of Fredholm operators, where $\bar{T}=\pi(T)$. One is the set of operators such that there exists an infinite dimensional and co-dimensional projection $P$ such that $\mathscr{A}_{T}=\pi(P)[\mathscr{L}(\mathscr{H}) / \mathscr{K}] \pi(P) \cong \mathscr{L}(\mathscr{H}) / \mathscr{K}$. Another is the set of operators such that $\mathscr{A}_{\bar{T}} \cong[\mathscr{L}(\mathscr{H}) / \mathscr{K}] \otimes \mathscr{K}$. For the last class, using 
$[37,2.5]$ repeatedly, we get a sequence of mutually orthogonal infinite dimensional and infinite co-dimensional projections $\left\{P_{n}\right\}$ such that $1=\sum_{n=1}^{\infty} P_{n}$ and $T=C+T_{0}$, where $C$ is a compact operator, $T_{0}$ is a noncompact operator and the entries $\left\{T_{i j}\right\}$ of $T_{0}$ with respect to the decomposition of the identity $1=\sum_{n=1}^{\infty} P_{n}$ are bounded operators such that $\left\|T_{i j}\right\| \rightarrow 0$ as either $i \rightarrow \infty$ or $j \rightarrow \infty$.

2. Real ranks of certain multiplier and corona algebras. We begin with a brief review on the construction of a Hilbert $C^{*}$-module $\mathscr{H}_{\mathscr{A}}$. Staring with a $C^{*}$-algebra $\mathscr{A}$, one can define an $\mathscr{A}$-valued inner product on the set of bounded sequences of elements of $\mathscr{A}$ by

$$
\left\langle\left\{a_{i}\right\},\left\{b_{i}\right\}\right\rangle=\sum_{i=1}^{\infty} a_{i}^{*} b_{i} .
$$

A Hilbert $C^{*}$-module $\mathscr{H}_{\mathscr{A}}$ consists of all sequences such that $\left\langle\left\{a_{i}\right\}\right.$, $\left.\left\{a_{i}\right\}\right\rangle$ exists as an element of $\mathscr{A}$. The $\mathscr{A}$-valued inner product $\langle\cdot, \cdot\rangle$ naturally induces a norm

$$
\|\cdot\|=\left\|\langle\cdot, \cdot\rangle^{1 / 2}\right\|
$$

on $\mathscr{H}_{\mathscr{A}}$. Referring to this norm, one can define bounded operators on $\mathscr{H}_{\mathscr{A}}$ in a similar way as on a Hilbert space $\mathscr{H}$. One can then define the adjoint operator $T^{*}$ for some bounded operator $T$ on $\mathscr{H}_{\mathscr{A}}$ by

$$
\left\langle T^{*}\left\{a_{i}\right\},\left\{b_{i}\right\}\right\rangle=\left\langle\left\{a_{i}\right\}, T\left\{b_{i}\right\}\right\rangle \text { for all }\left\{a_{i}\right\},\left\{b_{i}\right\} \in \mathscr{H}_{\mathscr{A}},
$$

where $T^{*}$ may not exist for some bounded operators. Let $\mathscr{L}\left(\mathscr{H}_{\mathscr{A}}\right)$ denote the set of all bounded operators on $\mathscr{H}_{\mathscr{A}}$ whose adjoint operators exist. Then $\mathscr{L}\left(\mathscr{H}_{\mathscr{A}}\right)$ is ${ }^{*}$-isomorphic to $M(\mathscr{A} \otimes \mathscr{K})$ as $C^{*}$-algebras. For each pair of elements $x$ and $y$ in $\mathscr{H}_{\mathscr{A}}$ one can define a bounded operator of rank one by

$$
\theta_{x, y}(z)=x\langle y, z\rangle \quad \text { for } z \in \mathscr{H}_{\mathscr{A}} .
$$

Let $\mathscr{K}\left(\mathscr{H}_{\mathscr{A}}\right)$ denote the closed linear span of all operators of rank one. Then $\mathscr{K}\left(\mathscr{H}_{\mathscr{A}}\right)$ is ${ }^{*}$-isomorphic to $\mathscr{A} \otimes \mathscr{K}$ as $C^{*}$-algebras. If $\mathscr{A}$ is the algebra of complex numbers, then $\mathscr{H}_{\mathscr{A}}$ is the separable Hilbert space $\mathscr{H}$. The reader is referred to [23] for more details.

More generally, we consider the pair of a $C^{*}$-algebra $\mathscr{A}$ and the associated multiplier algebra $M(\mathscr{A})$ where $\mathscr{A}$ may not be stable. Then $\mathscr{A}$ is a closed ideal of $M(\mathscr{A})$. We will frequently use the fact that $R R(M(\mathscr{A}))=0$ if and only if $M(\mathscr{A})$ has the (FS) property; and again if and only if the generalized Weyl-von Neumann theorem holds 
in $M(\mathscr{A})[37, \S 3]$. Obviously, $R R(M(\mathscr{A}) / \mathscr{A})=0$ if $R R(M(\mathscr{A}))$ $=0$.

The following Theorem (2.1) and Theorem (2.2) conclude that the generalized Weyl-von Neumann theorem holds in the multiplier algebras of many interesting $C^{*}$-algebras.

2.1. Theorem. Suppose that $\mathscr{A}$ is a $\sigma$-unital, nonunital, simple $C^{*}$-algebra with real rank zero and $M(\mathscr{A}) / \mathscr{A}$ is simple. Then the following hold:

(i) $R R(M(\mathscr{A}) / \mathscr{A})=0$.

(ii) $R R(M(\mathscr{A}))=0$ if and only if every projection in $M(\mathscr{A}) / \mathscr{A}$ lifts to a projection of $M(\mathscr{A})$.

One significant consequence is that in case $\mathscr{A}$ is a $\sigma$-unital (nonunital), purely infinite simple $C^{*}$-algebra, $R R(M(\mathscr{A}))=0$ iff $K_{1}(\mathscr{A})$ $=0$ (see Corollary (2.6)). It was unexpected that the triviality of $K_{1}(\mathscr{A})$, i.e., the connectedness of the unitary group of $\widetilde{\mathscr{A}}$ or equivalently the triviality of $K_{0}(M(\mathscr{A}) / \mathscr{A})$, has a lot to do with the 'amount' of projections in $M(\mathscr{A})$ (which is abundant enough so that $R R(M(\mathscr{A})$ ) $=0)$.

2.2. Theorem. Suppose that $\mathscr{A}$ is a $\sigma$-unital simple $C^{*}$-algebra with real rank zero and $M(\mathscr{A} \otimes \mathscr{K})$ has only one nontrivial closed ideal $\mathscr{J}_{0}$ strictly containing $\mathscr{A} \otimes \mathscr{K}$. Then the following hold:

(i) $R R\left(\mathscr{J}_{0} / \mathscr{A} \otimes \mathscr{K}\right)=0$, and $R R\left(M(\mathscr{A} \otimes \mathscr{K}) / \mathscr{J}_{0}\right)=0$.

(ii) $R R\left(\mathscr{J}_{0}\right)=0$ if and only if every projection in $\mathscr{J}_{0} / \mathscr{A} \otimes \mathscr{K}$ lifts.

(iii) $R R(M(\mathscr{A} \otimes \mathscr{K}) / \mathscr{A} \otimes \mathscr{K})=0$ if every projection in $M(\mathscr{A} \otimes \mathscr{K}) / \mathscr{J}_{0}$ lifts.

(iv) $R R(M(\mathscr{A} \otimes \mathscr{K}))=0$ if and only if every projection in $M(\mathscr{A} \otimes \mathscr{K}) / \mathscr{J}_{0}$ lifts and every projection in $\mathscr{J}_{0} / \mathscr{A} \otimes \mathscr{K}$ lifts.

Consequently, $R R(M(\mathscr{A} \otimes \mathscr{K}) / \mathscr{A} \otimes \mathscr{K})=0$ if $K_{1}\left(\mathscr{L}_{0}\right)=0 ; R R\left(\mathscr{J}_{0}\right)$ $=0$ if $K_{1}(\mathscr{A})=0$; and $R R(M(\mathscr{A} \otimes \mathscr{K}))=0$ if $K_{1}(\mathscr{A})=K_{1}\left(\mathscr{J}_{0}\right)=0$.

We need the following two technical lemmas in order to prove the above theorems. We denote the canonical mapping from $M(\mathscr{A} \otimes \mathscr{K})$ to $M(\mathscr{A} \otimes \mathscr{K}) / \mathscr{J}_{0}$ by $\phi$ and denote the identity of $M(\mathscr{A} \otimes \mathscr{K})$ by $1 \otimes 1$.

2.3. Lemma. Suppose that $\mathscr{A}$ is a $C^{*}$-algebra satisfying the hypotheses of Theorem (2.2). If $p$ is a projection in $M(\mathscr{A} \otimes \mathscr{K})$ but not 
in $\mathscr{J}_{0}$, then $\phi(1 \otimes 1) \lesssim \phi(p)$ in $M(\mathscr{A} \otimes \mathscr{K}) / \mathscr{J}_{0}$, and hence $\phi(p)$ is an infinite projection.

Proof. If $\mathscr{A}$ has enough traces to preserve the order of $D(\mathscr{A} \otimes \mathscr{K})$, then the techniques of comparing projections would work [38, 24]. However, it is not known whether there is any trace on such a $C^{*}$ algebra. Hence, we have to use the following technical construction.

Since $\mathscr{A}$ is $\sigma$-unital and simple, $\mathscr{A} \otimes \mathscr{K} \cong r \mathscr{A} r \otimes \mathscr{K}$ for any nonzero projection $r$ of $\mathscr{A}([9,2.8])$. Hence, we assume that $\mathscr{A}$ has a unit from now on.

Since $p$ generates $M(\mathscr{A} \otimes \mathscr{K})$ as a closed ideal, by [40, 2.3] there is an integer $n \geq 1$ such that $[1 \otimes 1] \leq n[p]$.

If $n=1$, then $1 \otimes 1 \lesssim p$. It follows that $\phi(1 \otimes 1) \lesssim \phi(p)$. If $n=2$, then there are two projections $p^{\prime}$ and $p^{\prime \prime}$ in $M(\mathscr{A} \otimes \mathscr{K})$ such that $p^{\prime} \sim p, p^{\prime \prime} \sim p, p^{\prime \prime} p^{\prime}=0$ and $1 \otimes 1 \lesssim p^{\prime}+p^{\prime \prime}$. It follows that

$$
p^{\prime}+p^{\prime \prime} \sim 1 \otimes 1 \text {. }
$$

Take an isometry $v_{0} \in M(\mathscr{A} \otimes \mathscr{K})$ such that

$$
v_{0} v_{0}^{*}=p^{\prime} p^{\prime \prime} \quad \text { and } \quad v_{0}^{*} v_{0}=1 \otimes 1 \text {. }
$$

Since $R R(\mathscr{A})=R R(\mathscr{A} \otimes \mathscr{K})=0([12,2.6])$, every hereditary $C^{*}$ subalgebra of $\mathscr{A} \otimes \mathscr{K}$ has an approximate identity consisting of projections $([5,2.7]$ and [27]). By [37, 1.2] there are sequences of mutually orthogonal projections $\left\{f_{i}^{\prime}\right\}$ and $\left\{f_{i}^{\prime \prime}\right\}$ in $\mathscr{A} \otimes \mathscr{K}$ such that

$$
\sum_{i=1}^{\infty} f_{i}^{\prime}=v_{0}^{*} p^{\prime} v_{0} \quad \text { and } \quad \sum_{i=1}^{\infty} f_{i}^{\prime \prime}=v_{0}^{*} p^{\prime \prime} v_{0}, \quad \text { where } f_{i}^{\prime} \sim f_{i}^{\prime \prime} \forall i \geq 1 .
$$

Clearly, $\left\{\sum_{i=1}^{n}\left(f_{i}^{\prime}+f_{i}^{\prime \prime}\right)\right\}$ constitutes an approximate identity of $\mathscr{A} \otimes$ $\mathscr{K}$ consisting of projections, while $\left\{\sum_{i=1}^{m} 1 \otimes e_{i i}\right\}$ is another approximate identity consisting of projections. By an argument of $\mathrm{G}$. A. Elliott in [21] there exist two subsequences $\left\{n_{k}\right\}$ and $\left\{m_{k}\right\}$ of integers and a unitary $v$ in $M(\mathscr{A} \otimes \mathscr{K})$ such that

$$
\begin{aligned}
\sum_{i=1}^{m_{1}} 1 \otimes e_{i i} & \leq v\left[\sum_{i=1}^{n_{1}}\left(f_{i}^{\prime}+f_{i}^{\prime \prime}\right)\right] v^{*} \leq \cdots \leq \sum_{i=1}^{m_{k}} 1 \otimes e_{i i} \\
& \leq v\left[\sum_{i=1}^{n_{k}}\left(f_{i}^{\prime}+f_{i}^{\prime \prime}\right)\right] v^{*} \leq \sum_{i=1}^{m_{k+1}} 1 \otimes e_{i i} \leq \cdots
\end{aligned}
$$

It is clear for any fixed $k$ that

$$
1 \otimes e_{11} \sim r_{1} \leq \sum_{i=n_{k}+1}^{n_{k+2}}\left(f_{i}^{\prime}+f_{i}^{\prime \prime}\right) \quad\left(\text { where } n_{0}=0\right) \text {. }
$$


Diagonalizing $r_{1}$ as in $[39,3.3]$ or $[42,1.1]$ with respect to the direct sum

$$
\left(\sum_{i=n_{k}+1}^{n_{k+2}} f_{i}^{\prime}\right)+\left(\sum_{i=n_{k}+1}^{n_{k+2}} f_{i}^{\prime \prime}\right),
$$

we have

$$
\begin{gathered}
r_{1} \sim r_{11}+r_{12}, \quad \text { where }\left[r_{12}\right] \leq\left[r_{11}\right] \leq\left[\sum_{i=n_{k}+1}^{n_{k+2}} f_{i}^{\prime}\right] \quad \text { and } \\
{\left[r_{12}\right] \leq\left[\sum_{i=n_{k}+1}^{n_{k+2}} f_{i}^{\prime \prime}\right]}
\end{gathered}
$$

Since

$$
\left[1 \otimes e_{11}\right] \leq\left[\sum_{i=n_{k+2}+1}^{n_{k+4}}\left(f_{i}^{\prime}+f_{i}^{\prime \prime}\right)\right]
$$

there is a projection $r_{12}^{\prime}$ such that

$$
r_{12} \sim r_{12}^{\prime} \leq \sum_{i=n_{k+2}+1}^{n_{k+4}}\left(f_{i}^{\prime}+f_{i}^{\prime \prime}\right) .
$$

Diagonalizing $r_{12}^{\prime}$ with respect to

$$
\left(\sum_{i=n_{k+2}+1}^{n_{k+4}} f_{i}^{\prime}\right)+\left(\sum_{i=n_{k+2}+1}^{n_{k+4}} f_{i}^{\prime \prime}\right)
$$

we have

$$
\begin{gathered}
r_{12}^{\prime} \sim r_{21}+r_{22}, \quad \text { where }\left[r_{22}\right] \leq\left[r_{21}\right] \leq\left[\sum_{i=n_{k+2}+1}^{n_{k+4}} f_{i}^{\prime}\right] \text { and } \\
{\left[r_{22}\right] \leq\left[\sum_{i=n_{k+2}+1}^{n_{k+4}} f_{i}^{\prime \prime}\right]}
\end{gathered}
$$

Then $2\left[r_{22}\right] \leq\left[r_{21}\right]+\left[r_{11}\right]$. Proceeding in this way, for any $l \geq 1$ we 
can find projections $\left\{r_{1}^{\prime}, r_{2}^{\prime}, \ldots, r_{l}^{\prime}, s_{l}\right\}$ in $\mathscr{A} \otimes \mathscr{K}$ such that

$$
\begin{gathered}
r_{j}^{\prime} \sim r_{j 1} \quad \text { for } 1 \leq j \leq l, \quad s_{l} \sim r_{l 2}, \quad\left[s_{l}\right] \leq\left[r_{l}^{\prime}\right] \leq \cdots \leq\left[r_{2}^{\prime}\right] \leq\left[r_{1}^{\prime}\right], \\
1 \otimes e_{11}=\sum_{j=1}^{l} r_{j}^{\prime}+s_{l}, \quad \sum_{j=1}^{l}\left[r_{j}^{\prime}\right] \leq\left[\sum_{i=n_{k}+1}^{n_{k+2 l}} f_{i}^{\prime}\right], \quad \text { and } \\
{\left[s_{l}\right] \leq\left[\sum_{j=n_{k}+1}^{n_{k+2 l}} f_{i}^{\prime \prime}\right]}
\end{gathered}
$$

Obviously, $l\left[s_{l}\right] \leq \sum_{j=1}^{l}\left[r_{j}^{\prime}\right]$.

Proceeding recursively, we can find a subsequence $\left\{n_{k}^{\prime}\right\}$ and write the identity $1 \otimes 1=\sum_{i=1}^{\infty} 1 \otimes e_{i i}$ as a sum of two terms:

$$
1 \otimes 1=\sum_{k=1}^{\infty} r_{k}+\sum_{k=1}^{\infty} s_{k}
$$

where $k\left[s_{k}\right] \leq\left[r_{k}\right]$ (since $r_{k}$ has a form $\sum_{j=1}^{l} r_{j}^{\prime}$ ), $s_{k} \lesssim \sum_{i=n_{k}^{\prime}+1}^{n_{k+1}^{\prime}} f_{i}^{\prime \prime}$ and $r_{k} \lesssim \sum_{i=n_{k}^{\prime}+1}^{n_{k}^{\prime}+1} f_{i}^{\prime}$. It is routine to show that

$$
\sum_{k=1}^{\infty} r_{k} \lesssim v_{0}^{*} p^{\prime} v_{0} \quad \text { and } \quad \sum_{k=1}^{\infty} s_{k} \lesssim v_{0}^{*} p^{\prime \prime} v_{0} .
$$

If $p_{0}=\sum_{k=1}^{\infty} s_{k}$ is a projection of $\mathscr{J}_{0}$, then $\phi(1 \otimes 1)=\phi\left(\sum_{k=1}^{\infty} r_{k}\right) \lesssim$ $\phi\left(p^{\prime}\right) \sim \phi(p)$.

If $p_{0}$ is not in $\mathscr{J}_{0}$, then by $[40,2.3]$ again we have $[1 \otimes 1] \leq$ $m\left[p_{0}\right]$. By construction, $m\left[\sum_{k=m+1}^{\infty} s_{k}\right] \leq\left[\sum_{k=m+1}^{\infty} r_{k}\right]$. It follows that $\phi(1 \otimes 1) \lesssim \phi\left(\sum_{k=m+1}^{\infty} r_{k}\right) \lesssim \phi\left(p^{\prime}\right) \sim \phi(p)$.

If $n>2$, we choose an integer $m$ such that $2^{m}>n$. Then $[1 \otimes 1] \leq$ $2^{m}[p]$. Repeating $m$ times the above argument for $n=2$, we will reach $\phi(1 \otimes 1) \lesssim \phi(p)$. The details are left to the reader.

REMARK. Actually, under the same hypotheses as in Lemma (2.3) we can prove a stronger conclusion: Every projection $p$ in $M(\mathscr{A} \otimes \mathscr{K})$ not in $\mathscr{J}_{0}$ is equivalent to $1 \otimes 1$. Since it takes more room for the technical details and also it is beyond the need of this article, we decided to give the proof elsewhere.

2.4. Lemma (cf. [12, 3.14] and [42, 2.3]). Suppose that $\mathscr{B}$ is a $C^{*}$-algebra and $\mathscr{I}$ is a closed ideal of $\mathscr{B}$. Then $R R(\mathscr{B})=0$ if and 
only if $R R(\mathscr{I})=0, R R(\mathscr{B} / \mathscr{I})=0$ and every projection in $\mathscr{B} / \mathscr{I}$ lifts to a projection in $\mathscr{B}$.

Proof. We give the following different proof for the direction 'if', since this proof contains new information about the lifting from $\mathscr{B} / \mathscr{I}$ to $\mathscr{B}$.

Assume that $R R(\mathscr{B} / \mathscr{I})=0$ and $R R(\mathscr{I})=0$ and every projection of $\mathscr{B} / \mathscr{I}$ lifts to a projection in $\mathscr{B}$. Let $x$ be a self-adjoint element in $\mathscr{B}$ and $\delta$ a positive number. We show that $x$ can be approximated in norm by positive elements with finite spectra.

Since $R R(\mathscr{B} / \mathscr{J})=0$, there exist real numbers $\left\{t_{i}\right\}$ and mutually orthogonal projections $\left\{\bar{p}_{i}\right\}$ in $\mathscr{B} / \mathscr{I}$ such that $\left\|\bar{x}-\sum_{i=1}^{m} t_{i} \bar{p}_{i}\right\|<\delta$, where $\bar{x}$ means the image of $x$ in $\mathscr{B} / \mathscr{I}$. Since every projection of $\mathscr{B} / \mathscr{I}$ lifts, the projections $\left\{\bar{p}_{i}\right\}$ lift to mutually orthogonal projections $\left\{p_{i}\right\}$ in $\mathscr{B}([37,2.5])$. But $\left\|x-\sum_{i=1}^{m} t_{i} p_{i}\right\|$ may not be small. We adjust the element $\sum_{i=1}^{n} t_{i} p_{i}$ so that $\left\|x-\sum_{i=1}^{n} t_{i} p_{i}\right\|$ can be arbitrarily small and hence $R R(\mathscr{B})=0$. By $[1,4.3]$, there is a self-adjoint element $a$ in $\mathscr{I}$ such that

$$
\left\|x-\sum_{i=1}^{m} t_{i} p_{i}-a\right\|=\left\|\bar{x}-\sum_{i=1}^{m} t_{i} \bar{p}_{i}\right\|<\delta .
$$

Set $p_{0}=1-\sum_{i=1}^{n} p_{i}$. Then $p_{0}$ is a projection in $\widetilde{\mathscr{I}}$. Since $R R(\mathscr{I})=$ 0 , for $0 \leq i \leq m$ we can choose an approximate identity $\left\{p_{i}\left(\lambda_{i}\right): \lambda_{i} \in\right.$ $\left.\Lambda_{i}\right\}$ of $p_{i} \mathscr{A} p_{i}$ consisting of projections. Then

$$
\left\{\sum_{i=1}^{m} p_{i}\left(\lambda_{i}\right):\left\{\lambda_{i}\right\} \in \Lambda_{0} \times \Lambda_{1} \times \cdots \times \Lambda_{m}\right\}
$$

constitutes an approximate identity of $\mathscr{I}$. Set $q_{0}=\sum_{i=1}^{m} p_{i}\left(\lambda_{i}\right)$ for some $\left\{\lambda_{i}\right\}$ such that $\left\|\left(1-q_{0}\right) a\right\|<\delta$. Then

$$
\begin{aligned}
& \left\|\left(1-q_{0}\right)\left(x-\sum_{i=1}^{m} t_{i} p_{i}\right)\right\| \\
& \quad \leq\left\|\left(1-q_{0}\right)\left(x-\sum_{i=1}^{m} t_{i} p_{i}-a\right)\right\|+\left\|\left(1-q_{0}\right) a\right\|<2 \delta,
\end{aligned}
$$

and hence

$$
\left\|\left(1-q_{0}\right) x q_{0}\right\|=\left\|\left(1-q_{0}\right)\left(\dot{x}-\sum_{i=1}^{m} t_{i} p_{i}\right) q_{0}\right\|<2 \delta
$$


and

$$
\left\|\left(1-q_{0}\right)\left(x-\sum_{i=1}^{m} t_{i} p_{i}\right)\left(1-q_{0}\right)\right\|<2 \delta .
$$

Thus

$$
\begin{aligned}
& \left\|x-q_{0} x q_{0}-\sum_{i=1}^{m} t_{i}\left(p_{i}-p_{i}\left(\lambda_{i}\right)\right)\right\| \\
& \quad \leq 2\left\|\left(1-q_{0}\right) x q_{0}\right\|+\left\|\left(1-q_{0}\right)\left(x-\sum_{i=1}^{m} t_{i} p_{i}\right)\left(1-q_{0}\right)\right\|<6 \delta .
\end{aligned}
$$

Since $R R(\mathscr{I})=0$, there is a self-adjoint element $f_{\delta}$ in $q_{0} \mathscr{I} q_{0}$ with a finite spectrum such that $\left\|q_{0} x q_{0}-f_{\delta}\right\|<\delta$.

Set $h_{\delta}=f_{\delta}+\sum_{i=1}^{m} t_{i}\left(p_{i}-p_{i}\left(\lambda_{i}\right)\right)$. Since $f_{\delta}, p_{1}-p_{1}\left(\lambda_{1}\right), p_{2}-$ $p_{2}\left(\lambda_{2}\right), \ldots$, and $p_{m}-p_{m}\left(\lambda_{m}\right)$ are mutually orthogonal, it is clear that $h_{\delta}$ has a finite spectrum. It follows from the above estimates that

$$
\left\|x-h_{\delta}\right\| \leq\left\|x-q_{0} x q_{0}-\sum_{i=1}^{m} t_{i}\left(p_{i}-p_{i}\left(\lambda_{i}\right)\right)\right\|+\left\|q_{0} x q_{0}-f_{\delta}\right\|<7 \delta .
$$

\subsection{Proof of Theorem (2.1) and Theorem (2.2).}

Proof. Since $\mathscr{A}$ is simple such that $R R(\mathscr{A})=0, M(A) / A$ is purely infinite by $[38,1.3]$ no matter $\mathscr{A}$ is stable or not. If $M(\mathscr{A}) / \mathscr{A}$ is simple, then $R R(M(\mathscr{A}) / \mathscr{A})=0$ by Theorem (1.2). By Lemma (2.4), $R R(M(\mathscr{A}))=0$ if and only if every projection in $M(\mathscr{A}) / \mathscr{A}$ lifts. Hence, Theorem (2.1) is clear.

Now we prove Theorem (2.2). By [38, 1.3] again, we see that $\mathscr{J}_{0} / \mathscr{A} \otimes \mathscr{K}$ is purely infinite and simple. It follows from Theorem (1.2) that $R R\left(\mathscr{J}_{0} / \mathscr{A} \otimes \mathscr{K}\right)=0$. By Lemma $(2.4), R R\left(\mathscr{J}_{0}\right)=0$ if and only if every projection in $\mathscr{J}_{0} / \mathscr{A} \otimes \mathscr{K}$ lifts. To prove $R R\left(M(\mathscr{A} \otimes \mathscr{K}) / \mathscr{J}_{0}\right)=0$, by Lemma (2.3) and Theorem (1.2), it suffices to prove that $M(\mathscr{A} \otimes \mathscr{K}) / \mathscr{J}_{0}$ is purely infinite and simple. By definition, it is sufficient to prove that for any nonzero positive element $\tilde{x}$ in $M(\mathscr{A} \otimes \mathscr{K}) / \mathscr{J}_{0}$ there exists an infinite projection $\tilde{p}$ in the hereditary $C^{*}$-subalgebra of $M(\mathscr{A} \otimes \mathscr{K}) / \mathscr{J}_{0}$ generated by $\tilde{x}$. Let $x$ be a positive element in $M(\mathscr{A} \otimes \mathscr{K})$ not in $\mathscr{J}_{0}$ such that $\phi(x)=\tilde{x}$. Since every hereditary $C^{*}$-subalgebra of $M(\mathscr{A} \otimes \mathscr{K})$ is the closed linear span of its projections $([38,1.1])$, there is a projection $p$ in $x M(\mathscr{A} \otimes \mathscr{K}) x$ not in $\mathscr{J}_{0}$. By Lemma $(2.3), \phi(p)$ is an infinite projection. Thus, (i) and (ii) are clear. 
To prove (iii), we consider the following short exact sequence:

$$
0 \rightarrow \mathscr{J}_{0} / \mathscr{A} \otimes \mathscr{K} \rightarrow M(\mathscr{A} \otimes \mathscr{K}) / \mathscr{A} \otimes \mathscr{K} \rightarrow M(\mathscr{A} \otimes \mathscr{K}) / \mathscr{J}_{0} \rightarrow 0,
$$

where we use the natural isomorphism

$$
(M(\mathscr{A} \otimes \mathscr{K}) / \mathscr{A} \otimes \mathscr{K}) /\left(\mathscr{J}_{0} / \mathscr{A} \otimes \mathscr{K}\right) \cong M(\mathscr{A} \otimes \mathscr{K}) / \mathscr{J}_{0} .
$$

Since $R R\left(M(\mathscr{A} \otimes \mathscr{K}) / \mathscr{J}_{0}\right)=0$ and $R R\left(\mathscr{J}_{0} / \mathscr{A} \otimes \mathscr{K}\right)=0$, it follows from Lemma (2.4) that $R R(M(\mathscr{A} \otimes \mathscr{K}) / \mathscr{A} \otimes \mathscr{K})=0$ if and only if every projection in $M(\mathscr{A} \otimes \mathscr{K}) / \mathscr{J}_{0}$ lifts to a projection in $M(\mathscr{A} \otimes \mathscr{K}) / \mathscr{A} \otimes \mathscr{K}$. If $\tilde{p}$ is any projection in $M(\mathscr{A} \otimes \mathscr{K}) / \mathscr{J}_{0}$, then $\tilde{p}$ lifts to a projection $p$ in $M(\mathscr{A} \otimes \mathscr{K})$ by hypothesis. Clearly, $\tilde{p}$ lifts to a projection $\pi(p)$ in $M(\mathscr{A} \otimes \mathscr{K}) / \mathscr{A} \otimes \mathscr{K}$. Hence, (iii) is clear.

It follows from (ii) that $R R\left(\mathscr{J}_{0}\right)=0$ if every projection of $\mathscr{J}_{0} / \mathscr{A} \otimes$ $\mathscr{K}$ lifts. It then follows from Lemma (2.4) that $R R(M(\mathscr{A} \otimes \mathscr{K}))=0$ if, in addition, every projection in $M(\mathscr{A} \otimes \mathscr{K}) / \mathscr{J}_{0}$ lifts. Since the projection lifting holds whenever $K_{1}$ is trivial ([37, §2]), the consequences are clear. This completes the proof.

Theorem (2.1) and Theorem (2.2) have included many interesting $C^{*}$-algebras. Let us first state a corollary and then provide some specific examples.

2.6. Corollary. Suppose that $\mathscr{A}$ is a $\sigma$-unital, purely infinite, simple $C^{*}$-algebra. Then the following hold:

(i) $R R(M(\mathscr{A} \otimes \mathscr{K}) / \mathscr{A} \otimes \mathscr{K})=0$.

(ii) $R R(M(\mathscr{A} \otimes \mathscr{K}))=0$ if and only if $K_{1}(\mathscr{A})=0$. In other words, the generalized Weyl-von Neumann theorem holds in $\mathscr{L}\left(\mathscr{H}_{\mathscr{A}}\right)$ if and only if $K_{1}(\mathscr{A})=0$.

Proof. Note if $\mathscr{A}$ is non-unital, then $\mathscr{A}$ is automatically stable by Theorem (1.2). We state the result in the form of stabilization to include the cases that $\mathscr{A}$ is unital.

By [40, 3.3], the generalized Calkin algebra $M(\mathscr{A} \otimes \mathscr{K}) / \mathscr{A} \otimes \mathscr{K}$ is purely infinite and simple. It follows from Theorem (1.2) that $R R(\mathscr{A})=0$ and $R R(M(\mathscr{A} \otimes \mathscr{K}) / \mathscr{A} \otimes \mathscr{K})=0$. By [37, §2], $K_{1}(\mathscr{A})=$ 0 if and only if every projection in $M(\mathscr{A} \otimes \mathscr{K}) / \mathscr{A} \otimes \mathscr{K}$ lifts. Now Theorem (2.1) implies the equivalence between ' $K_{1}(\mathscr{A})=0$ ' and $' R R(M(\mathscr{A} \otimes \mathscr{K}))=0$ '. 
2.7. EXAMPLES. (i) If $2 \leq n \leq \infty$, then $R R\left(M\left(\mathscr{O}_{n} \otimes \mathscr{K}\right)\right)=0$ (of course $R R\left(M\left(\mathscr{O}_{n} \otimes \mathscr{K}\right) / \mathscr{O}_{n} \otimes \mathscr{K}\right)=0$, too).

(ii) If $A$ is an irreducible matrix such that $\operatorname{det}(I-A)=0$, then $R R\left(M\left(\mathscr{O}_{A} \otimes \mathscr{K}\right) / \mathscr{O}_{A} \otimes \mathscr{K}\right)=0 \quad$ but $\quad R R\left(M\left(\mathscr{O}_{A} \otimes \mathscr{K}\right)\right) \neq 0$.

(iii) If $\mathscr{C}$ is the Calkin algebra, then $R R(M(\mathscr{C} \otimes \mathscr{K}) / \mathscr{A} \otimes \mathscr{K})=0$ but $R R(M(\mathscr{C} \otimes \mathscr{K})) \neq 0$.

(iv) If $\mathscr{A}$ is a type III factor, then $R R(M(\mathscr{A} \otimes \mathscr{K}))=0$.

In fact, $\mathscr{O}_{n}(2 \leq n \leq \infty)$ and $\mathscr{O}_{A}$, if $A$ is an irreducible matrix, are purely infinite and simple ([15]). It is well known that the Calkin algebra and a type III factor are purely infinite and simple. Hence, it follows from Proposition (1.4) that the stabilizations of these $C^{*}$ algebras are purely infinite and simple, too. It follows from Corollary (2.6) that the generalized Calkin algebras associated with these $C^{*}$ algebras have real rank zero.

It was proved ([15]) that $K_{1}\left(\mathscr{O}_{n}\right)=0$ for $2 \leq n \leq \infty$ and $K_{1}\left(\mathscr{O}_{A}\right) \neq$ 0 when $\operatorname{det}(I-A)=0$. It is well known that $K_{1}(\mathscr{C})$ is the group of integers and the $K_{1}$ of a type III factor is trivial. Thus, (i)-(iv) are clear from Corollary (2.6).

Theorem (2.1) and Theorem (2.2) have also included many 'finite' $C^{*}$-algebras in the sense of having trace(s). Using the terminology in [24], we say that $\mathscr{A}$ has a continuous scale if every trace on $\mathscr{A}$ extends to a finite trace on $M(\mathscr{A})$ and $\hat{1}(\tau)=\lim _{n \rightarrow \infty} \tau\left(e_{n}\right)$ is continuous on the state space $S_{u}$ of $K_{0}(\mathscr{A})$, where $u$ is an order unit of $K_{0}(\mathscr{A})$ and $\left\{e_{n}\right\}$ is a sequential increasing approximate identity of $\mathscr{A}$ consisting of projections (the existence of such $\left\{e_{n}\right\}$ is guaranteed by $[37,1.2])$.

2.8. ExAmple. If $\mathscr{A}$ is a $\sigma$-unital, nonunital, simple AF-algebra with a continuous scale (in particular, if $\mathscr{A}$ is a finite matroid algebra), then $R R(M(\mathscr{A}))=0$ [12].

In fact, since $K_{1}(\mathscr{A})=0$, then it follows from [10] or [37, §2] that every projection in $M(\mathscr{A}) / \mathscr{A}$ lifts. By [38, 1.1] and [24, §2], $M(\mathscr{A}) / \mathscr{A}$ is purely infinite and simple. Hence the conclusion follows from Theorem (1.2) and Theorem (2.1).

A (quasi)trace $\tau$ on $\mathscr{A}$ is said to be order-preserving if $\tau(p)<\tau(q)$ implies $[p] \leq[q]$, where $p$ and $q$ are two projections of $\mathscr{A}$. Each trace $\tau$ on $\mathscr{A}$ extends to a trace on $M(\mathscr{A} \otimes \mathscr{K})$ whose values can be $+\infty$, still denoted by $\tau$. Let $\mathscr{J}_{\tau}$ be the closed ideal of $M(\mathscr{A} \otimes \mathscr{K})$ generated by all projections whose trace values are not $+\infty$. 
2.9. Proposition (cf. $[40,3.6])$. If $\mathscr{A}$ is a unital $C^{*}$-algebra with real rank zero and an order-preserving (quasi)trace $\tau$, then every projection in $M(\mathscr{A} \otimes \mathscr{K})$ but not in $\mathscr{J}_{\tau}$ is equivalent to the identity $1 \otimes 1$, and hence $\mathscr{J}_{\tau}$ is the unique nontrivial closed ideal of $M(\mathscr{A} \otimes \mathscr{K})$ strictly containing $\mathscr{A} \otimes \mathscr{K}$.

Proof. If $p$ is a projection $p$ of $M(\mathscr{A} \otimes \mathscr{K})$ not in $\mathscr{J}_{\tau}$, then $\tau(p)=$ $+\infty$. By $[39, \S 4]$ there is a unitary $u$ in $M(\mathscr{A} \otimes \mathscr{K})$ such that $q=$ $u p u^{*}=\sum_{i=1}^{\infty} p_{i} \otimes e_{i i}$ where $\left\{p_{i}\right\}$ is a sequence of nonzero projections of $\mathscr{A}$. Of course $\tau(q)=+\infty$. Then we can choose a subsequence $\left\{n_{k}\right\}$ of positive integers such that $\tau\left(1 \otimes e_{k k}\right)<\tau\left(\sum_{i=n_{k-1}+1}^{n_{k}} p_{i} \otimes e_{i i}\right)$ for each $k \geq 1$ where $\left(k_{0}=0\right)$. Since $\tau$ is order-preserving, there is a partial isometry $v_{k}$ in $\mathscr{A} \otimes \mathscr{K}$ such that

$$
v_{k} v_{k}^{*}=1 \otimes e_{k k} \quad \text { and } \quad v_{k}^{*} v_{k}<\sum_{i=n_{k-1}+1}^{n_{k}} p_{i} \otimes e_{i i} \quad \text { for all } k \geq 1
$$

Set $w=\sum_{k=1}^{\infty} v_{k}$. Then $w$ is a partial isometry of $M(\mathscr{A} \otimes \mathscr{K})$ such that

$$
w w^{*}=1 \otimes 1 \quad \text { and } \quad w^{*} w<q .
$$

Since $K_{1}(M(\mathscr{A} \otimes \mathscr{K}))=0$ ([4]), it follows from [15] that $1 \otimes 1 \sim q$. Of course $1 \otimes 1 \sim p$.

Since $R R(\mathscr{A})=0$, it follows from $[12,2.10]$ that $R R(\mathscr{A} \otimes \mathscr{K})=$ 0 . By $[40, \S 2]$ every closed ideal of $M(\mathscr{A} \otimes \mathscr{K})$ is generated by its projections. Therefore, $\mathscr{J}_{\tau}$ is the only proper closed ideal strictly containing $\mathscr{A} \otimes \mathscr{K}$.

In [36], the author proved that if $\mathscr{A}$ is a $\sigma$-unital AF algebra or $\mathscr{A}$ is the tensor product of a finite factor with $\mathscr{K}$, then every hereditary $C^{*}$-subalgebra, and hence every closed ideal, of $M(\mathscr{A})$ has a trivial $K_{1}$-group. Combining this result with Theorem (2.2) and Proposition (2.9), we have the following:

2.10. ExAmple. If $\mathscr{A}$ is a $\sigma$-unital, simple AF algebra with a unique trace (up to multiples), in particular if $\mathscr{A}$ is a separable matroid algebra (of course the trace is order-preserving), then $R R(M(\mathscr{A} \otimes \mathscr{K}))=0$. Example (2.8) together with Example (2.10) include a result $[12,3.21]$.

2.11. EXAMPLE. It is well known that there is an order-preserving trace on a type $\mathrm{II}_{1}$ factor $\mathscr{M}$ and hence on $\mathscr{M} \otimes \mathscr{K}$. Thus, $R R(M(\mathscr{M} \otimes \mathscr{K}))=0$. 
It was proved ([14]) that for a dense subset of irrational numbers the corresponding irrational rotation algebras have FS (and hence have real rank zero by $[12,2.6])$. In addition, there is a unique quasitrace $\tau$ on an irrational rotation algebra $\mathscr{A}_{\theta}$, which is order-preserving ([6, 5.3.2] or [31]-[33]).

2.12. EXAMPLE. If $\mathscr{A}_{\theta}$ is an irrational rotation $C^{*}$-algebra such that $R R\left(\mathscr{A}_{\theta}\right)=0$, then $R R\left(\mathscr{J}_{\tau} / \mathscr{A}_{\theta} \otimes \mathscr{K}\right)=0$ and $R R\left(M\left(\mathscr{A}_{\theta} \otimes \mathscr{K}\right) / \mathscr{J}_{\tau}\right)$ $=0$ (by Theorem (2.2) and Proposition (2.9)). However, $R R\left(M\left(\mathscr{A}_{\theta} \otimes \mathscr{K}\right)\right) \neq 0$, since $K_{1}\left(\mathscr{A}_{\theta}\right) \neq 0$ ([4] or [31]-[33]).

Here a question remains unanswered: Is $R R\left(M\left(\mathscr{A}_{\theta} \otimes \mathscr{K}\right) / \mathscr{A}_{\theta} \otimes \mathscr{K}\right)$ zero?

It was proved ([8], [12], [27]) that the Bunce-Deddens algebras defined in [2] have real rank zero. There is a unique tracial state $\tau$ on a Bunce-Deddens algebra ([3]), and the tracial state $\tau$ is orderpreserving $([6,5.1])$.

2.13. EXAMPLE. If $\mathscr{B}$ is one of the Bunce-Deddens algebras, then $R R\left(M(\mathscr{B} \otimes \mathscr{K}) / \mathscr{J}_{\tau}\right)=0$ and $R R\left(\mathscr{J}_{\tau} / \mathscr{B} \otimes \mathscr{K}\right)=0$ (by Theorem (2.2) and Proposition (2.9)). Moreover, $R R(M(\mathscr{B} \otimes \mathscr{K}) / \mathscr{B} \otimes \mathscr{K})=0$, since $K_{1}\left(\mathscr{J}_{\tau}\right)=0$. But $R R(M(\mathscr{B} \otimes \mathscr{K})) \neq 0$, since $K_{1}(\mathscr{B}) \neq 0$.

To see the last two sentences above, we need a recent result of L. G. Brown (via private communication) that the multiplier algebra of a $C^{*}$-algebra stably isomorphic to one of the Bunce-Deddens algebras has a connected unitary group and hence has a trivial $K_{1}$. Using this fact, one can prove that $K_{1}\left(\mathscr{J}_{\tau}\right)=0$, and then every projection in $M(\mathscr{B} \otimes \mathscr{K}) / \mathscr{J}_{\tau}$ lifts by $[37, \S 2]$.

Now we show that $K_{1}\left(\mathscr{J}_{\tau}\right)=0$. If $r$ is any projection in $\mathscr{J}_{\tau}$ not in $\mathscr{B} \otimes \mathscr{K}$, then $M(r(\mathscr{B} \otimes \mathscr{K}) r)=r M(\mathscr{B} \otimes \mathscr{K}) r=r \mathscr{J}_{\tau} r$ and $r \mathscr{J}_{\tau} r$ generates $\mathscr{J}_{\tau}$ as a closed ideal. Hence $K_{1}(M(r(\mathscr{B} \otimes \mathscr{K}) r))=K_{1}\left(\mathscr{J}_{\tau}\right)$. Since $\mathscr{B}$ is simple $([2])$, it follows from $[9,2.8]$ that $r(\mathscr{B} \otimes \mathscr{K}) r$ is stably isomorphic to $\mathscr{B}$. Now applying the result of L. G. Brown mentioned above, we conclude that $K_{1}(M(r(\mathscr{B} \otimes \mathscr{K}) r))=0$, and hence $K_{1}\left(\mathscr{J}_{\tau}\right)=0$, as desired.

\section{Relevant matters and more examples.}

3.1. A new look at the classical Weyl-von Neumann theorem. The $C^{*}$-algebraic approach in the last section provides new insights into the classical Calkin algebra 
Reducing to the case $\mathscr{A}=\mathscr{K}$, we see that the classical Weyl-von Neumann theorem is equivalent to the facts that $R R(\mathscr{K})=0$ and $K_{1}(\mathscr{K})=0$. From this, the scene behind the Weyl-von Neumann theorem becomes quite clear. We sketch the following purely $C^{*}$ algebraic proof without using any techniques arising from the underline Hilbert space $\mathscr{H}$ nor the fact that $\mathscr{L}(\mathscr{H})$ is a von Neumann algebra.

Outline of the proof. (Necessity): Assume that the Weyl-von Neumann theorem holds (i.e., $R R(\mathscr{L}(\mathscr{H}))=0$ ). Then $R R(\mathscr{K})=0$, since $\mathscr{K}$ is a closed ideal of $\mathscr{L}(\mathscr{H})$. On the other hand, since $R R(\mathscr{L}(\mathscr{H}))=0$, it follows from $[37,2.2]$ that every projection of $\mathscr{L}(\mathscr{H}) / \mathscr{K}$ lifts to a projection in $\mathscr{L}(\mathscr{H})$. Thus, $K_{1}(\mathscr{K})=0[37$, 2.10].

(Sufficiency). By $[39, \S 4]$, every projection of $\mathscr{L}(\mathscr{H})$ is unitarily equivalent to a projection with a diagonal form whose off diagonal entries are 0's and diagonal entries are either 0 or 1 . Moreover, every projection in $\mathscr{L}(\mathscr{H})$ not in $\mathscr{K}$ is Murray-von Neumann equivalent to the identity. Since $K_{1}(\mathscr{K})=0$, every projection in $\mathscr{L}(\mathscr{H}) / \mathscr{K}$ lifts to a projection in $\mathscr{L}(\mathscr{H})$. It follows that every nonzero projection in $\mathscr{L}(\mathscr{H}) / \mathscr{K}$ is infinite. By [38, 1.1], every nonzero hereditary $C^{*}$-subalgebra of $\mathscr{L}(\mathscr{H})$ is the closed linear span of its projections. Hence, $\mathscr{L}(\mathscr{H}) / \mathscr{K}$ is purely infinite and simple. It follows from Theorem (1.2) that $R R(\mathscr{L}(\mathscr{H}) / \mathscr{K})=0$. We conclude from Lemma (2.3) that $R R(\mathscr{L}(\mathscr{H}))=0$. Hence, the Weyl-von Neumann theorem holds $([37,3.1])$.

A strategy often used in proving that ' $R R(M(\mathscr{A}))=0$ ' is to show that the hereditary $C^{*}$-subalgebra $(x M(\mathscr{A}) x)^{-}$has an approximate identity consisting of projections for any positive element $x$ of $M(\mathscr{A})$. In case $\mathscr{A}$ is a $\sigma$-unital, purely infinite, simple $C^{*}$-algebra, $R R(M(\mathscr{A} \otimes \mathscr{K}))=0$ if and only if $K_{1}(\mathscr{A})=0$ (Corollary (2.6)). From the following theorem we will see what ' $K_{1}(\mathscr{A})=0$ ', or equivalently ' $R R(M(\mathscr{A} \otimes \mathscr{K}))=0$ ' really means in terms of hereditary $C^{*}$-subalgebras of $M(\mathscr{A} \otimes \mathscr{K})$. Here a hereditary $C^{*}$-subalgebra $\mathscr{B}$ of $M(\mathscr{A} \otimes \mathscr{K})$ is said to be essentially unital if $\pi(\mathscr{B})$ is a unital hereditary $C^{*}$-subalgebra of $M(\mathscr{A} \otimes \mathscr{K}) / \mathscr{A} \otimes \mathscr{K}$.

3.2. TheOREM. Suppose that $\mathscr{A}$ is a $\sigma$-unital, purely infinite, simple $C^{*}$-algebra. Then the following hold: 
(i) Every nonzero, $\sigma$-unital, hereditary $C^{*}$-subalgebra $\mathscr{B}$ of $M(\mathscr{A} \otimes \mathscr{K})$ is either stable or essentially unital.

(ii) If $\mathscr{B}$ is stable, then $\mathscr{B}$ is either *-isomorphic to $\mathscr{A} \otimes \mathscr{K}$ or *-isomorphic to $M(\mathscr{A} \otimes \mathscr{K}) \otimes \mathscr{K}$.

(iii) If $\mathscr{B}$ is unital, then $\mathscr{B}$ is either *-isomorphic to $M(\mathscr{A} \otimes \mathscr{K})$ or $r \mathscr{A} r$ for some projection $r$ in $\mathscr{A}$.

(iv) If $\mathscr{B} \not \subset \mathscr{A} \otimes \mathscr{H}$ is essentially unital, but nonunital, and if $K_{1}(\mathscr{A})=0$, then $\mathscr{B}$ is *-isomorphic to a hereditary $C^{*}$-subalgebra of $M(\mathscr{A} \otimes \mathscr{K})$ of the form

$$
\left(\begin{array}{cc}
p M(\mathscr{A} \otimes \mathscr{K}) p & p(\mathscr{A} \otimes \mathscr{K})(1-p) \\
(1-p)(\mathscr{A} \otimes \mathscr{K}) p & (1-p)(\mathscr{A} \otimes \mathscr{K})(1-p)
\end{array}\right),
$$

where $p$ is a projection in $\mathscr{B}$ but not in $\mathscr{A} \otimes \mathscr{K}$. Moreover, by Theorem (1.2), $(1-p)(\mathscr{A} \otimes \mathscr{K})(1-p)$ is either $*_{\text {-isomorphic to } \mathscr{A} \otimes \mathscr{K}}$ or *isomorphic to $r \mathscr{A} r$ for some projection $r$ in $\mathscr{A}$.

Proof. Let $\mathscr{B}$ be any $\sigma$-unital, hereditary $C^{*}$-subalgebra of $M(\mathscr{A} \otimes \mathscr{K})$.

(i), (ii) and (iii). If $\mathscr{B} \subset \mathscr{A} \otimes \mathscr{K}$, then $\mathscr{B}$ is either unital or stable by Theorem (1.2). If $\mathscr{B}$ is unital, then $\mathscr{B}=r_{1}(\mathscr{A} \otimes \mathscr{K}) r_{1}$ for some projection $r_{1}$ in $\mathscr{A} \otimes \mathscr{K}$. By Theorem (1.2) and a standard argument for simple $C^{*}$-algebras (see [5, 3.12.1], for example), we see that $\mathscr{B} \cong r \mathscr{A} r$ for some nonzero projection $r$ in $\mathscr{A}$. If $\mathscr{B}$ is stable, then $\mathscr{B} \cong \mathscr{A} \otimes \mathscr{K}$. In fact, since $\mathscr{A} \otimes \mathscr{K}$ is purely infinite and simple, it is clear that $\mathscr{B}$ is purely infinite and simple. Since both $\mathscr{A} \otimes \mathscr{K}$ and $\mathscr{B}$ are $\sigma$-unital, $[9,2.8]$ applies.

We assume that $\mathscr{B}$ is not contained in $\mathscr{A} \otimes \mathscr{K}$. Set $\overline{\mathscr{B}}=\pi(\mathscr{B})$. Then $\overline{\mathscr{B}}$ is $\sigma$-unital. Since $M(\mathscr{A} \otimes \mathscr{K}) / \mathscr{A} \otimes \mathscr{K}$ is simple and purely infinite $([40,3.3]), \overline{\mathscr{B}}$ is either unital or stable by Theorem (1.2). If $\mathscr{B}$ is unital, the unit $p$ of $\mathscr{B}$ is a projection of $M(\mathscr{A} \otimes \mathscr{K})$ equivalent to the identity by $[\mathbf{4 0}, 3.3]$. It follows that $\mathscr{B} \cong M(\mathscr{A} \otimes \mathscr{K})$.

If $\mathscr{B}$ is not essentially unital, then $\overline{\mathscr{B}}$ is stable. We show that $\mathscr{B}$ is stable, and that $\mathscr{B} \cong M(\mathscr{A} \otimes \mathscr{K}) \otimes \mathscr{K}$. Since $\mathscr{B}$ is $\sigma$-unital, $\mathscr{B}$ is the hereditary $C^{*}$-subalgebra of $M(\mathscr{A} \otimes \mathscr{K})$ generated, as a hereditary $C^{*}$-subalgebra, by a single positive element $x$ in $\mathscr{B}$ but not in $\mathscr{A} \otimes$ $\mathscr{K}$. As in the proof of Theorem (1.2), we define a sequence of positive functions $f_{n}$, and then construct an increasing sequential approximate identity $\left\{e_{n}\right\}$ of $\mathscr{B}$ such that $e_{n} e_{m}=e_{n}$ if $m>n$. Consider two sequences of hereditary $C^{*}$-subalgebras of $\mathscr{B}$, say $\left\{\mathscr{B}_{n}\right\}$ and $\left\{\mathscr{C}_{n}\right\}$, as follows: 
$\mathscr{B}_{n}=\operatorname{her}\left(e_{2 n-1}-e_{2 n-2}\right), \quad \mathscr{C}_{n}=\operatorname{her}\left(e_{2 n}-e_{2 n-1}\right), \quad n=1,2,3, \ldots$

It is clear that $\mathscr{B}_{n}$ and $\mathscr{B}_{m}$ are mutually orthogonal and $\mathscr{C}_{n}$ and $\mathscr{C}_{m}$ are mutually orthogonal if $n \neq m$. Moreover, the union of the $\mathscr{B}_{n}$ 's and the $\mathscr{C}_{n}$ 's generates $\mathscr{B}$ as a hereditary $C^{*}$-subalgebra.

We claim that either $\left\{\pi\left(\mathscr{B}_{n}\right)\right\}$ or $\left\{\pi\left(\mathscr{C}_{n}\right)\right\}$ has an infinite nonzero subsequence. Otherwise, there would exist a positive integer $n_{0}$ such that $\pi\left(\left(1-e_{n_{0}}\right) \mathscr{B}\left(1-e_{n_{0}}\right)\right)=0$. By the construction of $\left\{e_{n}\right\}$, it would imply that $\pi(x)$ is invertible in $M(\mathscr{A} \otimes \mathscr{K}) / \mathscr{A} \otimes \mathscr{K}$, and hence $\pi(\mathscr{B})$ would be $M(\mathscr{A} \otimes \mathscr{K}) / \mathscr{A} \otimes \mathscr{K}$. This contradicts the hypotheses. We can assume that $\pi\left(\mathscr{B}_{n}\right)$ is nonzero for $n \geq 1$. Hence $\mathscr{B}_{n} \not \subset \mathscr{A} \otimes \mathscr{K}$ for $n \geq 1$. By $[38,1.1]$, there exists a projection $p_{n}$ in $\mathscr{B}_{n} \backslash \mathscr{A} \otimes \mathscr{K}$ for $n \geq 1$. It is obvious that $p_{n} p_{m}=0$ if $n \neq m$. Set $q=\sum_{n=1}^{\infty} p_{n}$. It is routine to show that $q$ is a projection in $M(\mathscr{B})$. It follows from [40, 3.3] that $p_{n} \sim 1$ for each $n \geq 1$. Thus $q \mathscr{B} q$ is a stable hereditary $C^{*}$-subalgebra of $\mathscr{B}$. Since $q \mathscr{B} q \not \subset \mathscr{A} \otimes \mathscr{K}$ and $\pi(\mathscr{B})$ is simple $([40,2.3$ and 3.3]), of course $q \mathscr{B} q$ generates $\mathscr{B}$ as a closed ideal. Now Lemma (1.1) applies, and hence $\mathscr{B}$ is stable. Actually, $\mathscr{B} \cong M(\mathscr{A} \otimes \mathscr{K}) \otimes \mathscr{K}$ by [40, 3.3]. We have proved (i), (ii) and (iii).

(iv) From now on we assume that $K_{1}(\mathscr{A})=0$ and $\mathscr{\mathscr { B }}$ has the unit $\bar{p}$. It is clear that $\mathscr{B} \cap(\mathscr{A} \otimes \mathscr{K})$ is a hereditary $C^{*}$-subalgebra of $\mathscr{A} \otimes \mathscr{K}$, and hence $\mathscr{B} \cap(\mathscr{A} \otimes \mathscr{K})$ has real rank zero by Theorem (1.2). Since $K_{1}(\mathscr{A})=0$, it follows from $[9,2.8]$ that $K_{1}(\mathscr{B} \cap(\mathscr{A} \otimes \mathscr{K}))=0$. By $[37,2.12]$ there exists a projection $p$ in $\mathscr{B}$ such that $\pi(p)=\bar{p}$. It is easily verified that $(1-p) \mathscr{B} p \cup p \mathscr{B}(1-p) \cup(1-p) \mathscr{B}(1-p) \subset \mathscr{A} \otimes \mathscr{K}$. Moreover, since $p$ is in $\mathscr{B}$ but not in $\mathscr{A} \otimes \mathscr{K}$, we see from [40, 3.3] that $p \sim 1$. On the other hand, $(1-p) \mathscr{B}(1-p)$ is either unital or stable, and hence is either isomorphic to $r \mathscr{A} r$ for some projection in $\mathscr{A}$ or isomorphic to $\mathscr{A} \otimes \mathscr{K}$ (by (iii)). Now we clearly see that $\mathscr{B}$ is *-isomorphic to the matrix form.

The following corollary provides a new condition equivalent to ' $K_{1}(\mathscr{A})=0$ '.

3.3. Corollary. If $\mathscr{A}$ is a $\sigma$-unital, purely infinite, simple $C^{*}$ algebra, then the following are equivalent:

(i) $R R(M(\mathscr{A} \otimes \mathscr{K}))=0$ (iff $K_{1}(\mathscr{A})=0$, by Corollary $(2.6)$ ).

(ii) Every essentially unital, non-unital, hereditary $C^{*}$-subalgebra of $M(\mathscr{A} \otimes \mathscr{K})$ has an approximate identity consisting of projections.

Proof. It is clear that (i) implies (ii). By Theorem (3.2)(i), every non-essentially unital hereditary $C^{*}$-subalgebra of $M(\mathscr{A} \otimes \mathscr{K})$ is 
stable, and hence automatically has an approximate identity consisting of projections. Hence, (ii) implies (i).

3.4. A sequence of corona algebras with real rank zero. Starting with a $C^{*}$-algebra $\mathscr{A}$ such that $R R(\mathscr{A})=0$ and $M(\mathscr{A}) / \mathscr{A}$ is purely infinite and simple, we can construct a sequence of purely infinite, simple corona algebras, by recursively setting

$$
C_{(n)}(\mathscr{A})=M\left(C_{(n-1)}(\mathscr{A}) \otimes \mathscr{K}\right) / C_{(n-1)}(\mathscr{A}) \otimes K
$$

with

$$
\begin{aligned}
& C_{(1)}(\mathscr{A})=M(\mathscr{A}) / \mathscr{A}, \\
& C_{(2)}(\mathscr{A})=M([M(\mathscr{A}) / \mathscr{A}] \otimes \mathscr{K}) /[M(\mathscr{A}) / \mathscr{A}] \otimes \mathscr{K}, \ldots .
\end{aligned}
$$

By $[38,1.1]$ and $[40,3.3]$, for each $n \geq 1, C_{(n)}(\mathscr{A})$ is a purely infinite, simple corona algebra. Hence

$$
R R\left(C_{(n)}(\mathscr{A})\right)=0 \quad \text { for } n \geq 1 \text { by Theorem (1.2). }
$$

3.5. Proposition. If $\mathscr{A}$ is a stable $C^{*}$-algebra, then for $m \geq 1$

$$
\begin{aligned}
& K_{1}\left(C_{(2 m-1)}(\mathscr{A})\right) \cong K_{0}\left(C_{(2 m)}(\mathscr{A})\right) \cong K_{0}(\mathscr{A}), \quad \text { and } \\
& K_{0}\left(C_{(2 m-1)}(\mathscr{A})\right) \cong K_{1}\left(C_{(2 m)}(\mathscr{A})\right) \cong K_{1}(\mathscr{A}) .
\end{aligned}
$$

In particular, if $K_{1}(\mathscr{A})=0$, then $K_{1}\left(C_{(2 m)}(\mathscr{A})\right)=0$; if $K_{0}(\mathscr{A})=0$, then $K_{1}\left(C_{(2 m-1)}(\mathscr{A})\right)=0$ for $m \geq 1$; if $K_{1}(\mathscr{A})=K_{0}(\mathscr{A})=0$, then $K_{1}\left(C_{(m)}(\mathscr{A})\right)=0$ for all $m \geq 1$.

Proof. This follows by repeatedly applying the two well-known isomorphisms:

$$
\begin{aligned}
& K_{1}(\mathscr{A}) \cong K_{0}(M(\mathscr{A} \otimes \mathscr{K}) / \mathscr{A} \otimes \mathscr{K}) \quad \text { and } \\
& K_{0}(\mathscr{A}) \cong K_{1}(M(\mathscr{A} \otimes \mathscr{K}) / \mathscr{A} \otimes \mathscr{K}),
\end{aligned}
$$

which are consequences of the six-term exact sequence of $K$-theory corresponding to the short exact sequence:

$0 \rightarrow \mathscr{A} \otimes \mathscr{K} \rightarrow M(\mathscr{A} \otimes \mathscr{K}) / \mathscr{A} \otimes \mathscr{K} \rightarrow M(\mathscr{A} \otimes \mathscr{K}) \rightarrow 0$

3.6. Corollary. Suppose that $\mathscr{A}$ is a $\sigma$-unital, nonunital, purely infinite, simple $C^{*}$-algebra. Then

$$
\begin{array}{ll}
R R\left(M\left(C_{(2 m-1)}(\mathscr{A}) \otimes \mathscr{K}\right)\right) \neq 0 & \text { if } K_{0}(\mathscr{A}) \neq 0 \\
R R\left(M\left(C_{(2 m)}(\mathscr{A}) \otimes \mathscr{K}\right)\right) \neq 0, & \text { if } K_{1}(\mathscr{A}) \neq 0 .
\end{array}
$$


Proof. Since $\mathscr{A}$ is automatically stable by Theorem (1.2), it follows from $[40,3.3]$ that $C_{(m)}(\mathscr{A})$ is purely infinite and simple, and hence $R R\left(C_{(m)}(\mathscr{A})\right)=0$ for all $m \geq 1$. Now the conclusion follows from Corollary (2.6).

\subsection{ExAMPLE. Since $K_{1}\left(\mathscr{O}_{2}\right)=K_{0}\left(\mathscr{O}_{2}\right)=0$ ([15]),}

$$
R R\left(M\left(C_{(n)}\left(\mathscr{O}_{2} \otimes \mathscr{K}\right) \otimes \mathscr{K}\right)\right)=0 \quad \text { for } n \geq 1
$$

(by Proposition (3.5) and Corollary (2.6)).

3.8. Example. If $\mathscr{A}$ is a type III factor, then

$$
R R\left(M\left(C_{(n)}(\mathscr{A} \otimes \mathscr{K}) \otimes \mathscr{K}\right)\right)=0
$$

$\left(\right.$ since $K_{1}(\mathscr{A})=K_{0}(\mathscr{A})=0$ ).

3.9. ExAMPLe. If $\mathscr{A}$ is a finite separable, nonunital matroid algebra, then

$$
R R\left(C_{(m)}(\mathscr{A})\right)=0 \quad \text { and } \quad R R\left(M\left(C_{(2 m-1)}(\mathscr{A}) \otimes \mathscr{K}\right)\right)=0,
$$

but $R R\left(M\left(C_{(2 m)}(\mathscr{A}) \otimes \mathscr{K}\right)\right) \neq 0 \quad(m \geq 1)$.

In fact, ' $K_{1}(\mathscr{A})=0$ ' follows from [21, $\S 2$ ]. Recursively, we have

$$
\begin{aligned}
K_{1}\left(C_{(1)}(\mathscr{A})\right)=K_{1}(M(\mathscr{A}) / \mathscr{A}) & =0 \\
K_{0}\left(C_{(2)}(\mathscr{A})\right) \cong K_{1}\left(C_{(1)}(\mathscr{A})\right)=0, \quad K_{1}\left(C_{(3)}(\mathscr{A})\right) \cong K_{0}\left(C_{(2)}(\mathscr{A})\right) & =0 \\
K_{0}\left(C_{(4)}(\mathscr{A})\right) \cong K_{1}\left(C_{(3)}(\mathscr{A})\right)=0, \quad K_{1}\left(C_{(5)}(\mathscr{A})\right) \cong K_{0}\left(C_{(4)}(\mathscr{A})\right) & =0
\end{aligned}
$$

Hence $K_{0}\left(C_{(2 m)}(\mathscr{A})\right) \cong K_{1}\left(C_{(2 m-1)}(\mathscr{A})\right)=0$ for $m \geq 1$. Similarly, we have

$$
K_{0}\left(C_{(2 m-1)}(\mathscr{A})\right) \cong K_{0}(M(\mathscr{A}) / \mathscr{A}) \cong K_{1}\left(C_{(2 m)}(\mathscr{A})\right) \neq 0 \quad \text { for } m \geq 1 \text {. }
$$

4. $K_{1}$ of infinite simple $C^{*}$-algebras. The $K_{*}$-groups of a unital, purely infinite, simple $C^{*}$-algebra $\mathscr{A}$ have been described by J. Cuntz in [15]. The $K_{0}$-group of $\mathscr{A}$ is identified with the set of Murray-von Neumann equivalence classes of nonzero projections in $\mathscr{A}$, which forms a group in this special situation. The $K_{1}$-group of $\mathscr{A}$ is identified with $U(\mathscr{A}) / U_{0}(\mathscr{A})$, where $U(\mathscr{A})$ is the unitary group of $\mathscr{A}$ and $U_{0}(\mathscr{A})$ is the path component of $U(\mathscr{A})$ containing the identity.

In this section, we give a description for the $K_{1}$-group of an arbitrarily purely infinite simple $C^{*}$-algebra $\mathscr{A}$ (not necessarily $\sigma$-unital).

We say that two unitaries $u$ and $v$ are homotopic, denoted by ' $u \sim v$ ', if $u$ and $v$ are in the same path component of the unitary group. 
4.1. Lemma. Assume that $\mathscr{A}$ is a nonunital $C^{*}$-algebra with an approximate identity consisting of projections. If $u$ is a unitary of $\widetilde{A}$, then there exist a projection $q$ in $\mathscr{A}$ and a unitary $u_{1}+(1-q)$ homotopic to $u$ in the unitary group of $\widetilde{\mathscr{A}}$, where $u_{1}$ is a unitary of $q \mathscr{A} q$. Moreover, if $p$ is a projection of $\mathscr{A}$, we can choose the projection $q$ such that $p<q$.

Proof. It is clear that $u$ can be written as $\lambda 1+z$ for some element $z$ in $\mathscr{A}$ and a complex number $\lambda$ with $|\lambda|=1$. Since $\mathscr{A}$ has an approximate identity consisting of projections, we can choose a projection $q_{0}$ in $\mathscr{A}$ such that both

$$
\left\|\left(1-q_{0}\right) p\right\| \text { and }\left\|q_{0} u q_{0}+\lambda\left(1-q_{0}\right)-u\right\|
$$

are small enough. By $[21,2.1]$, there exists a unitary $v$ in $\widetilde{\mathscr{A}}$ close to the identity in norm such that $p \leq v q_{0} v^{*}$. Replacing $q_{0}$ by $v q_{0} v^{*}=$ $q$, we obtain that $p \leq q$ and $\|q u q+\lambda(1-q)-u\|$ is small. Since $\mathscr{A}$ is nonunital, we can choose the $q$ such that $p<q$. It follows that $q u q+\lambda(1-q)$ is an invertible element of $\widetilde{\mathscr{A}}$. It is clear that $q u q+\lambda(1-q)$ is homotopic, in the group of invertible elements of $\widetilde{\mathscr{A}}$, to both $u$ and the unitary $u^{\prime}$ occurring in the polar decomposition of $q u q+\lambda(1-q)$. Clearly, $u^{\prime}$ has the form $u_{1}+(1-q)$, and $u$ is homotopic to $u_{1}+(1-q)$ in the unitary group of $\widetilde{\mathscr{A}}$.

4.2. Lemma. Assume that $\mathscr{A}$ is a nonunital $C^{*}$-algebra with an approximate identity consisting of projections. If $\{u(t): 0 \leq t \leq 1\}$ is a path of unitaries in $\widetilde{\mathscr{A}}$ such that $u(0)=1$ and $u(1)=u_{0}+(1-p)$ for some projection $p$ in $\mathscr{A}$, then there exist a projection $q>p$ in $\mathscr{A}$ and another path of unitaries $\{v(t)+(1-q): 0 \leq t \leq 1\}$ such that $v(0)=q$ and $v(1)=u_{0}+(q-p)$, where $\{v(t): 0 \leq t \leq 1\}$ is a path of unitaries of $q \mathscr{A} q$.

Proof. Let $\varepsilon$ be a positive number less than $1 / 6$. Take a subdivision of the interval $[0,1]: 0=t_{0}<t_{1}<t_{2}<\cdots, t_{n-1}<t_{n}=1$, such that $\left\|u\left(t_{i}\right)-u\left(t_{i-1}\right)\right\|<\varepsilon$ for $i=1,2, \ldots, n$. It is clear that $u\left(t_{i}\right)=$ $\lambda_{i} 1+z_{i}$, where $\lambda_{i}$ is a complex number with $\left|\lambda_{i}\right|=1$ and $z_{i}$ is an element in $\mathscr{A}$ for $1 \leq i \leq n$. Since $\mathscr{A}$ has an approximate identity consisting of projections, there exists a projection $q$ such that $p<q$ and $\left\|q u\left(t_{i}\right) q+\lambda_{i}(1-q)-u\left(t_{i}\right)\right\|<\varepsilon$ for $1 \leq i \leq n$. Then $q u\left(t_{i}\right) q+\lambda_{i}(1-q) \quad(1 \leq i \leq n)$ are all invertible elements of $\widetilde{\mathscr{A}}$, and hence $q u\left(t_{i}\right) q(1 \leq i \leq n)$ are invertible in $q \mathscr{A} q$. Since 
$\left\|q u\left(t_{i}\right) q-q u\left(t_{i-1}\right) q\right\| \leq\left\|u\left(t_{i}\right)-u\left(t_{i-1}\right)\right\|<\varepsilon$, the $q u\left(t_{i}\right) q$ 's are all in the same path component of invertible elements of $q \mathscr{A} q$. Let $v\left(t_{i}\right)$ be the unitary of $q \mathscr{A} q$ occurring in the polar decomposition of $q u\left(t_{i}\right) q$ for $1 \leq i \leq n$. It is clear that the $v\left(t_{i}\right)$ 's are in the same path component of the unitary group of $q \mathscr{A} q$. Moreover, for $1 \leq i \leq n, u\left(t_{i}\right)$ is homotopic to $v\left(t_{i}\right)+(1-q)$ in the unitary group of $\widetilde{\mathscr{A}}$. Let $\{v(t): 0 \leq$ $t \leq 1\}$ be a path of unitaries of $q \mathscr{A} q$ joining the $v\left(t_{i}\right)$ 's. Since $p<q$, it is clear from construction that $v(0)=q$ and $v(1)=u_{0}+(q-p)$.

4.3. Lemma. If $\mathscr{A}$ is a simple stable $C^{*}$-algebra with a nonzero projection, then $\mathscr{A}$ has an approximate identity consisting of projections.

Proof. The conclusion follows from $[9,2.8]$.

Now we give a description of the $K_{*}$-groups of an arbitrary purely infinite simple $C^{*}$ - algebra, which slightly extends a result of [15].

4.4. THeOREM. If $\mathscr{A}$ is a purely infinite simple $C^{*}$-algebra (not necessarily $\sigma$-unital), and if $p$ is any fixed nonzero projection of $\mathscr{A}$, then

$$
K_{0}(\mathscr{A}) \cong D(p \mathscr{A} p) \quad \text { and } \quad K_{1}(\mathscr{A})=U(p \mathscr{A} p) / U_{0}(p \mathscr{A} p) .
$$

Here $D(p \mathscr{A} p)$ is the set of equivalence classes of nonzero projections in $p \mathscr{A} p$, which is actually a group ([15]).

Proof. First, identify $\mathscr{A}$ and $\mathscr{A} \otimes e_{11}$. Then $p \mathscr{A} p$ is identified with $p \mathscr{A} p \otimes e_{11}$.

Since $\mathscr{A}$ is a purely infinite simple $C^{*}$-algebra, so is $\mathscr{A} \otimes \mathscr{K}$ by Proposition 1.4. It is routine that every projection $q$ of $\mathscr{A} \otimes \mathscr{K}$ is close to a projection in $\mathscr{A} \otimes M_{n}$ in norm for some $n \geq 1$, and hence $q$ is unitarily equivalent to a projection of $\mathscr{A} \otimes M_{n}$. It follows from a standard argument (see [5, 3.12.10], for example) that $q$ is equivalent to a subprojection of $p$. It is clear that $D(\mathscr{A} \otimes \mathscr{K})$ is bijectively identified with $D(p \mathscr{A} p)$. By [15, 1.4 and 1.5], $K_{0}(p \mathscr{A} p) \cong D(p \mathscr{A} p)$. Since $D(\mathscr{A} \otimes \mathscr{K})$ is identified with $D(p \mathscr{A} p), K_{0}(\mathscr{A}) \cong D(p \mathscr{A} p)$.

Of course, our main job is to describe $K_{1}(\mathscr{A})$. To show that $K_{1}(\mathscr{A}) \cong U(p \mathscr{A} p) / U_{0}(p \mathscr{A} p)$ it suffices the following [44]:

(i) Every unitary element of $(\mathscr{A} \otimes \mathscr{K})^{+}$, the $C^{*}$-algebra obtained by joining an identity to $\mathscr{A} \otimes \mathscr{K}$, is homotopic to a unitary with the form $u_{0}+\left(1-p_{0}\right)$ in the unitary group of $(\mathscr{A} \otimes \mathscr{K})^{+}$, where $p_{0}$ is an 
infinite subprojection of $p$ such that $p \lesssim p-p_{0}$ and $u_{0}$ is a unitary of $p_{0} \mathscr{A} p_{0}$.

(ii) If $p_{0}$ is an infinite subprojection of $p$ such that $p \lesssim p-p_{0}$ and $u_{0}+\left(1-p_{0}\right)$ is an unitary in the path component of unitaries of $(\mathscr{A} \otimes \mathscr{K})^{+}$containing the identity, then $u_{0}+\left(p-p_{0}\right)$ is homotopic to the identity $p$ of $p \mathscr{A} p$ in the unitary group of $p \mathscr{A} p$.

Since $\mathscr{A} \otimes \mathscr{K}$ is purely infinite and simple, $\mathscr{A} \otimes \mathscr{K}$ has an approximate identity consisting of projections by Lemma (4.3). If $u$ is any unitary element of $(\mathscr{A} \otimes \mathscr{K})^{+}$, by Lemma (4.1) $u$ is homotopic to a unitary element $u_{1}+(1-q)$ in the unitary group of $(\mathscr{A} \otimes \mathscr{K})^{+}$, where $q$ is a projection of $\mathscr{A} \otimes \mathscr{K}$ such that $p<q$ and $u_{1}$ is a unitary of $q(\mathscr{A} \otimes \mathscr{K}) q$. We can assume that $q$ is in $\mathscr{A} \otimes M_{n}$ for some $n \geq 1$.

To prove (i), we need to show that $u_{1}+(1-q)$ is homotopic to a unitary with the form $u_{0}+\left(1-p_{0}\right)$ for some projection $p_{0}<p$ such that $p \lesssim p-p_{0}$. Let $q_{1}$ be a projection in $\mathscr{A} \otimes \mathscr{K}$ such that $q<q_{1}$ and $p \lesssim q_{1}-q$. It is clear that such $q_{1}$ exists. Then there exist partial isometries $w_{1}$ and $w_{2}$ in $q_{1}(\mathscr{A} \otimes \mathscr{K}) q_{1}$ such that

$$
\begin{gathered}
w_{1} w_{1}^{*}=q, \quad w_{1}^{*} w_{1} \leq q_{1}-q, \quad w_{2} w_{2}^{*}=w_{1}^{*} w_{1} \quad \text { and } \\
w_{2}^{*} w_{2}=p_{0}<p .
\end{gathered}
$$

It is clear that we can assume that $p \sim p^{\prime}<p-p_{0}$. Repeating one of the fundamentals $([15,1.7])$ of the $K_{1}$ twice, we obtain that $u_{1}+$ $\left(q_{1}-q\right), w_{1}^{*} u_{1} w_{1}+\left(q_{1}-w_{1}^{*} w_{1}\right)$ and $w_{2}^{*} w_{1}^{*} u_{1} w_{1} w_{2}+\left(q_{1}-w_{2}^{*} w_{2}\right)$ are all in the same path component of the unitary group of $q_{1}(\mathscr{A} \otimes \mathscr{H}) q_{1}$. Set $u_{0}=w_{2}^{*} w_{1}^{*} u_{1} w_{1} w_{2}$. Then $u_{0}$ is a unitary of $p_{0} \mathscr{A} p_{0}$ and $u_{1}+$ $(1-q)$ is homotopic to $u_{0}+\left(1-p_{0}\right)$ in the unitary group of $(\mathscr{A} \otimes \mathscr{K})^{+}$. Hence (i) follows.

Now we show (ii). Let $u_{0}+\left(1-p_{0}\right)$ be a unitary homotopic to the identity in the unitary group of $(\mathscr{A} \otimes \mathscr{K})^{+}$, where $p<p-p_{0}$ and $u_{0}$ is a unitary of $p_{0} \mathscr{A} p_{0}$. By Lemma (4.2), there is a projection $q>p$ in $\widetilde{\mathscr{A}} \otimes \mathscr{K}$ and a path of unitaries $\{v(t): 0 \leq t \leq 1\}$ of $q(\mathscr{A} \otimes \mathscr{K}) q$ such that

$$
v(0)=q \quad \text { and } \quad v(1)=u_{0}+\left(q-p_{0}\right),
$$

where we can assume that $q-p_{0}$ is infinite. Take a partial isometry $w$ in $\mathscr{A} \otimes \mathscr{K}$ such that

$$
w w^{*} \geq q-p_{0} \quad \text { and } \quad w^{*} w=p-p_{0} .
$$

Set $w_{1}=w+p_{0}$. Then

$$
w_{1} w_{1}^{*}=q_{1} \geq q, \quad w_{1}^{*} w_{1}=p \quad \text { and } \quad w_{1} p_{0}=p_{0} w_{1}=p_{0} .
$$


Set $v_{1}(t)=v(t)+\left(q_{1}-q\right)$ for $0 \leq t \leq 1$. Then $v_{1}(0)=q_{1}$ and $v(1)=u_{0}+\left(q_{1}-p_{0}\right)$. Set $w(t)=w_{1}^{*} v_{1}(t) w_{1}$ for each $0 \leq t \leq 1$.Then it is easily verified that $\{w(t): 0 \leq t \leq 1\}$ is a path of unitaries in $p(\mathscr{A} \otimes \mathscr{K}) p\left(\cong p \mathscr{A} p \otimes e_{11}\right)$ such that $w(0)=w_{1}^{*} v_{1}(0) w_{1}=p$ and $w(1)=w_{1}^{*} v_{1}(1) w_{1}=u_{0}+\left(p-p_{0}\right)$. Therefore (ii) holds.

Acknowledgment. The author wishes to thank the referee for his brief but very helpful suggestions which called his attention to several misprints in the manuscript.

\section{REFERENCES}

[1] C. A. Akemann, G. K. Pedersen and J. Tomiyama, Multipliers of $C^{*}$-algebra, J. Funct. Anal., 13 (1973), 277-301.

[2] J. Bunce and J. Deddens, A family of simple $C^{*}$-algebras related to weighted shift operators, J. Funct. Anal., 19 (1975), 13-24.

[3] _ , $C^{*}$-algebras generated by weighted shifts, Indiana Univ. Math. J., 23 (1973), 257-271.

[4] B. Blackadar, $K$-theory for Operator Algebras, Springer-Verlag, New York Berlin Heidelberg London Paris Tokyo 1987.

[5] _ Notes on the structure of projections in simple algebras, Semesterbericht Funktionalanalysis, Tubingen, Wintersemester 1982/83.

[6] - Comparison theory for simple $C^{*}$-algebras, Operator Algebras and Applications, LMS Lecture Notes, no 135, Cambridge University Press, 1988.

[7] B. Blackadar and J. Cuntz, The structure of stable algebraically simple $C^{*}$ algebras, Amer. J. Math., 104 (1982), 813-822.

[8] B. Blackadar and A. Kumjian, Skew products of relations and structure of simple $C^{*}$-algebras, Math. Z., 189 (1985), 55-63.

[9] L. G. Brown, Stable isomorphism of hereditary subalgebras of $C^{*}$-algebras, $\mathrm{Pa}$ cific J. Math., 71 (1977), 335-348.

[10] _- Extensions of AF algebras: the projection lifting problem, Operator Algebras and Applications, Proc. Sympos. Pure Math., vol. 38, Amer. Math. Soc., Providence, RI, 1981, Part I, 175-176.

[11] _ Semicontinuity and multipliers of $C^{*}$-algebras, Canad. J. Math., 40 (1988), 865-988.

[12] L. G. Brown and G. K. Pedersen, $C^{*}$-algebras of real rank zero, J. Funct. Anal., 99 (1991), 131-149.

[13] R Busby, Double centralizers and extensions of $C^{*}$-algebras, Trans. Amer. Math. Soc., 132 (1968), 79-99.

[14] M.-D. Choi and G. A. Elliot, Density of self-adjoint elements with finite spectrum in an irrational rotation $C^{*}$-algebra, Math. Scand., (to appear).

[15] J. Cuntz, K-theory for certain $C^{*}$-algebras, Ann. of Math., 131 (1981), 181197.

[16] _ Simple $C^{*}$-algebras generated by isometries, Comm. Math. Phys., 57 (1977), 173-185.

[17] J. Cuntz and N. Higson, Kuiper's theorem for Hilbert modules, Operator Algebras and Mathematical Physics, AMS, Vol. 62, Proceedings of a Summer conference, June, 17-21, 1985. 
[18] J. Cuntz and W. Krieger, A class of $C^{*}$-algebras and topological Markov chains, Invent. Math., 56 (1980), 251-268.

[19] J. Dixmier, On some $C^{*}$-algebras considered by Glimm, J. Funct. Anal., 1 (1967), 182-203.

[20] E. G. Effros, Dimensions and $C^{*}$-algebras, CBMS Regional Conference Series in Mathematics, No. 46, A.M.S., Providence, 1981.

[21] G. A. Elliott, Derivations of matroid $C^{*}$-algebras II, Ann. of Math., 100 (1974), 407-422.

[22] _ The ideal structure of multiplier algebra of an AF algebra, C. R. Math. Rep. Acad. Sci. Canada, 9 (1987), 225-230.

[23] G. G. Kasparov, Hilbert $C^{*}$-modules: Theorems of Stinespring and Voiculescu, J. Operator Theory, 4 (1980), 133-150.

[24] H. Lin, On ideals of multiplier algebras of simple AF $C^{*}$-algebras, Proc. Amer. Math. Soc., 104 (1988), 239-244.

[25] J. A. Mingo, $K$-theory and multipliers of stable $C^{*}$-algebras, Trans. Amer. Math. Soc., 299 (1987), 397-412.

[26] G. J. Murphy, Diagonality in $C^{*}$-algebras, Math. Z., 199 (1988), 279-284.

[27] G. K. Pedersen, The linear span of projections in simple $C^{*}$-algebras, J. Operator Theory, 4 (1980), 289-296.

[28] — SAW $^{*}$-algebras and corona $C^{*}$-algebras, contributions to non-commutative topology, J. Operator Theory, 15 (1986), 15-32.

[29] _ , $C^{*}$-algebras and Their Automorphism Groups, 1979, Academic Press, London New York San Francisco.

[30] C. L. Olsen and G. K. Pedersen, Corona $C^{*}$-algebras and their applications to lifting problems, Math. Scand., (to appear).

[31] M. Rieffel, $C^{*}$-algebras associated with irrational rotations, Pacific J. Math., 93 (1981), 415-429.

[32] _ Dimension and stable rank in the $K$-theory of $C^{*}$-algebras, Proc. London Math. Soc., 46 (1983), 301-333.

[33] — The cancellation theorem for projective modules over irrational rotation algebras, Proc. London Math. Soc., 47 (1983), 285-302.

[34] D. Voiculescu, A non-commutative Weyl-von Neumann theorem, Rev. Roumaine Math. Pour et Appl., 21 (1976), 97-113.

[35] $\mathrm{H}$. Weyl, Uber beschrankte quadratischen formen deren differentz vollstetigist, Rend. Circ. Mat. Palermo, 27 (1909), 373-392.

[36] S. Zhang, Trivial $K_{1}$-flow of AF algebras and finite von Neumann algebras, J. Funct. Anal., 92 (1990), 77-91.

[37] _ , $K_{1}$-groups, quasidiagonality and interpolation by multiplier projections, Trans. Amer. Math. Soc., 325 (1991), 793-818.

[38] _ On the structure of projections and ideals of corona algebras, Canad. J. Math., 41 (1989), 721-742.

[39] _ Diagonalizing projections in the multiplier algebras and matrices over a $C^{*}$-algebra, Pacific J. Math., 145 (1990), 181-200.

[40] _ A Riesz decomposition property and ideal structure of multiplier algebras, J. Operator Theory, (to appear).

[41] __ Certain $C^{*}$-algebras with real rank zero and their corona and multiplier algebras, Part II, K-Theory, (to appear).

[42] __, $C^{*}$-algebras with real rank zero and the internal structure of their corona and multiplier algebras, Part III, Canad. J. Math., 62 (1990), 159-190.

[43] _ Certain $C^{*}$-algebras with real rank zero and their corona and multiplier algebras, Part IV, Int. J. Math. (to appear). 
[44] S. Zhang, On the homotopy type of the unitary group and the Grassmann space of purely infinite simple $C^{*}$-algebras, $K$-Theory, (to appear).

Received June 1, 1990 and in revised form June 27, 1991. Partially supported by a grant from the National Science Foundation. Some results in this paper were presented at the 17th Annual Canadian Symposium on Operator Algebras and Operator Theory, held at University of Toronto during May 22 - May 26, 1989.

UNIVERSITY OF CINCINNATI

CinCINNATI, OH 45221-0025 



\section{PACIFIC JOURNAL OF MATHEMATICS EDITORS}

V. S. VARADARAJAN (Managing Editor)

University of California

Los Angeles, CA 90024-1555

vsv@math.ucla.edu

Herbert Clemens

University of Utah

Salt Lake City, UT 84112

clemens@math.utah.edu

F. Michael Christ

University of California

Los Angeles, CA 90024-1555

christ@math.ucla.edu

THOMAS ENRIGHT

University of California, San Diego

La Jolla, CA 92093

tenright@ucsd.edu
Nicholas ERcolani

University of Arizona

Tucson, AZ 85721

ercolani@math.arizona.edu

R. FINN

Stanford University

Stanford, CA 94305

finn@gauss.stanford.edu

VAUGHAN F. R. JoNES

University of California

Berkeley, CA 94720

vfr@math.berkeley.edu

STEVEN KeRCKHOFF

Stanford University

Stanford, CA 94305

spk@gauss.stanford.edu
C. C. MOORE

University of California

Berkeley, CA 94720

Martin ScharlemanN

University of California

Santa Barbara, CA 93106

mgscharl@henri.ucsb.edu

Harold Stark

University of California, San Diego

La Jolla, CA 92093

\begin{tabular}{|c|c|c|c|c|}
\hline \multicolumn{5}{|c|}{ ASSOCIATE EDITORS } \\
\hline R. ARENS & $\begin{array}{l}\text { E. F. BECKENBACH } \\
(1906-1982)\end{array}$ & B. H. NeumanN & $\begin{array}{c}\text { F. WoLF } \\
(1904-1989)\end{array}$ & K. YoshID \\
\hline \multicolumn{5}{|c|}{ SUPPORTING INSTITUTIONS } \\
\hline \multicolumn{2}{|c|}{ UNIVERSITY OF ARIZONA } & \multicolumn{3}{|c|}{ UNIVERSITY OF OREGON } \\
\hline \multicolumn{2}{|c|}{ UNIVERSITY OF BRITISH COLUMB } & \multicolumn{3}{|c|}{$\begin{array}{l}\text { UNIVERSITY OF SOUTHERN C } \\
\text { STANFORD UNIVERSITY }\end{array}$} \\
\hline \multicolumn{2}{|c|}{ CALIFORNIA INSTITUTE OF TECHNOLOGY } & \multirow{2}{*}{\multicolumn{3}{|c|}{$\begin{array}{l}\text { STANFORD UNIVERSITY } \\
\text { UNIVERSITY OF HAWAII }\end{array}$}} \\
\hline \multicolumn{2}{|c|}{ UNIVERSITY OF CALIFORNIA } & & & \\
\hline \multicolumn{2}{|c|}{ MONTANA STATE UNIVERSITY } & \multicolumn{3}{|c|}{ UNIVERSITY OF TOKYO } \\
\hline \multicolumn{2}{|c|}{ JNIVERSITY OF NEVADA, RENO } & \multicolumn{3}{|c|}{ UNIVERSITY OF UTAH } \\
\hline \multicolumn{2}{|c|}{ NEW MEXICO STATE UNIVERSITY } & \multicolumn{3}{|c|}{ WASHINGTON STATE UNIVERSITY } \\
\hline \multicolumn{2}{|c|}{ OREGON STATE UNIVERSITY } & \multicolumn{3}{|c|}{ UNIVERSITY OF WASHINGTON } \\
\hline
\end{tabular}




\section{PACIFIC JOURNAL OF MATHEMATICS}

Volume $155 \quad$ No. $1 \quad$ September 1992

Characterization of modular correspondences by geometric properties 1 Allan Russell AdLer

Representations of convex nondentable sets

SPIROS ARGYROS and IRENE DELIYANNI

Isomorphisms of spaces of continuous affine functions

CHO-Ho CHU and HENRY BRUCE COHEN

Universal classes of Orlicz function spaces

FRANCISCO LUIS HERNÁNDEZ RODRÍGUEZ and CESAR RUIZ

Asymptotic behavior of the curvature of the Bergman metric of the thin 99 domains

KANG-TAE KIM

Quadratic central polynomials with derivation and involution

CHARLES PHILIP LANSKI

Nonsplit ring spectra and products of $\beta$-elements in the stable homotopy of Moore spaces

\section{JIN KUN LIN}

Orientation and string structures on loop space

DENNIS MCLAUGHLIN

Homomorphisms of Bunce-Deddens algebras

\section{CORNEL PASNICU}

Certain $C^{*}$-algebras with real rank zero and their corona and multiplier 169 algebras. Part I

SHUANG ZHANG

Correction to: "On the density of twistor elementary states"

Michael G. Eastwood and A. M. Pilato 